\title{
The Maunder minimum (1645-1715) was indeed a grand minimum: A reassessment of multiple datasets
}

\author{
Ilya G. Usoskin ${ }^{1,2}$, Rainer Arlt ${ }^{3}$, Eleanna Asvestari ${ }^{1}$, Ed Hawkins ${ }^{6}$, Maarit Käpylä ${ }^{7}$, Gennady A. Kovaltsov ${ }^{4}$ \\ Natalie Krivova $^{5}$, Michael Lockwood ${ }^{6}$, Kalevi Mursula ${ }^{1}$, Jezebel O’Reilly ${ }^{6}$, Matthew Owens ${ }^{6}$, Chris J. Scott ${ }^{6}$, \\ Dmitry D. Sokoloff ${ }^{8,9}$, Sami K. Solanki ${ }^{5,10}$, Willie Soon ${ }^{11}$, and José M. Vaquero ${ }^{12}$ \\ ${ }^{1}$ ReSoLVE Centre of Excellence, University of Oulu, 90014, Finland \\ 2 Sodankylä Geophysical Observatory, University of Oulu, 90014, Finland \\ Leibniz Institute for Astrophysics Potsdam, An der Sternwarte 16, 14482 Potsdam, Germany \\ Ioffe Physical-Technical Institute, 194021 St. Petersburg, Russia \\ Max Planck Institute for Solar System Research, Justus-von-Liebig-Weg 3, 37077 Göttingen, Germany \\ ${ }^{6}$ Department of Meteorology, University of Reading, UK \\ ReSoLVE Centre of Excellence, Department of Computer Science, PO BOX 15400, Aalto University, 00076 Aalto, Finland \\ Moscow State University, 119991 Moscow, Russia \\ IZMIRAN, Moscow, Russia \\ 10 School of Space Research, Kyung Hee University, Yongin, 446-701 Gyeonggi, Republic of Korea \\ 11 Harvard-Smithsonian Center for Astrophysics, Cambridge, MA, USA \\ 12 Departamento de Física, Universidad de Extremadura, Mérida (Badajoz), 10003 Cáceres, Spain
}

Received 2 June 2015 / Accepted 12 July 2015

\begin{abstract}
Aims. Although the time of the Maunder minimum (1645-1715) is widely known as a period of extremely low solar activity, it is still being debated whether solar activity during that period might have been moderate or even higher than the current solar cycle \#24. We have revisited all existing evidence and datasets, both direct and indirect, to assess the level of solar activity during the Maunder minimum.

Methods. We discuss the East Asian naked-eye sunspot observations, the telescopic solar observations, the fraction of sunspot active days, the latitudinal extent of sunspot positions, auroral sightings at high latitudes, cosmogenic radionuclide data as well as solar eclipse observations for that period. We also consider peculiar features of the Sun (very strong hemispheric asymmetry of the sunspot location, unusual differential rotation and the lack of the K-corona) that imply a special mode of solar activity during the Maunder minimum.

Results. The level of solar activity during the Maunder minimum is reassessed on the basis of all available datasets.

Conclusions. We conclude that solar activity was indeed at an exceptionally low level during the Maunder minimum. Although the exact level is still unclear, it was definitely lower than during the Dalton minimum of around 1800 and significantly below that of the current solar cycle \#24. Claims of a moderate-to-high level of solar activity during the Maunder minimum are rejected with a high confidence level.
\end{abstract}

Key words. Sun: activity - sunspots - solar-terrestrial relations - history and philosophy of astronomy

\section{Introduction}

In addition to the dominant 11-year Schwabe cycle, solar activity varies on the centennial time scale (Hathaway 2010). It is a common present-day paradigm that the Maunder minimum (MM), occurring during the interval 1645-1715 (Eddy 1976), was a period of greatly suppressed solar activity called a grand minimum. Grand minima are usually considered as periods of greatly suppressed solar activity corresponding to a special state of the solar dynamo (Charbonneau 2010). Of special interest is the so-called core MM (1645-1700) when cyclic sunspot activity was barely visible (Vaquero \& Trigo 2015). Such grand minima are known from the indirect evidence provided by the cosmogenic isotopes ${ }^{14} \mathrm{C}$ and ${ }^{10} \mathrm{Be}$ data for the Holocene to occur sporadically, with the Sun spending on average one-sixth of the time in such a state (Usoskin et al. 2007). However, the MM is the only grand minimum covered by direct solar (and some relevant terrestrial) observations. It therefore forms a benchmark for other grand minima.
Other periods of reduced activity during the last centuries, such as the Dalton minimum (DM) at the turn of 19th century, the Gleissberg minimum around 1900, or the weak present solar cycle \#24, are also known, but they are typically not considered to be grand minima (Schüssler et al. 1997; Sokoloff 2004). However, the exact level of solar activity in the 17th century remains somewhat uncertain (e.g. Vaquero \& Vázquez 2009; Vaquero et al. 2011; Clette et al. 2014), leaving room for discussion and speculation. For example, there have been several suggestions that sunspot activity was moderate or even high during the core MM (1645-1700), being comparable to or even exceeding the current solar cycle \#24 (Schove 1955; Gleissberg et al. 1979; Cullen 1980; Nagovitsyn 1997; Ogurtsov et al. 2003; Nagovitsyn et al. 2004; Volobuev 2004; Rek 2013; Zolotova \& Ponyavin 2015). Some of these suggestions were based on a mathematical synthesis using empirical rules in a way similar to Schove (1955) and Nagovitsyn (1997) and therefore are not true reconstructions. Some others used a re-analysis of the direct 
data series (Rek 2013; Zolotova \& Ponyavin 2015) and provide claimed assessments of the solar variability. While earlier suggestions have been convincingly rebutted by Eddy (1983), the most recent ones are still circulating. If such claims were true, then the MM would not be a grand minimum. This would potentially cast doubts upon the existence of any grand minimum, including those reconstructed from cosmogenic isotopes.

There are indications that the underlying solar magnetic cycles still operated during the MM (Beer et al. 1998; Usoskin et al. 2001), but at the threshold level as proposed already by Maunder (1922):

It ought not to be overlooked that, prolonged as this inactivity of the Sun certainly was, yet few stray spots noted during "the seventy years' death" - 1660, 1671, 1684, $1695,1707,1718$ (we are, however, less certain about the exact timings of these activity maxima) - correspond, as nearly as we can expect, to the theoretical dates of maximum ... If I may repeat the simile which I used in my paper for Knowledge in 1894, "just as in a deeply inundated country, the loftiest objects will still raise their heads above the flood, and a spire here, a hill, a tower, a tree there, enable one to trace out the configuration of the submerged champaign", to the above mentioned years seem be marked out as the crests of a sunken spot-curve.

The nature of the MM is of much more than purely academic interest. A recent analysis of cosmogenic isotope data revealed a $10 \%$ chance that MM conditions would return within 50 years of now (Lockwood 2010; Solanki \& Krivova 2011; Barnard et al. 2011). It is therefore important to accurately describe and understand the MM, since a future grand minimum is expected to have significant implications for space climate and space weather.

Here we present a compilation of observational and historical facts and evidence showing that the MM was indeed a grand minimum of solar activity and that the level of solar activity was very low, much lower than that during the DM as well as in the present cycle \#24. In Sect. 2 we revisit sunspot observations during the MM. In Sect. 3 we analyze indirect proxy records of solar activity, specifically aurorae borealis and cosmogenic isotopes. In Sect. 4 we discuss consequences of the MM for solar dynamo and solar irradiance modeling. Conclusions are presented in Sect. 5.

\section{Sunspot observation in the 17 th century}

Figure 1 shows different estimates of sunspot activity around the MM, quantified in terms of the annual group sunspot number (GSN) $R_{G}$. The GSN has recently been corrected as a result of newly uncovered data or corrections of earlier errors being applied (see details in Vaquero et al. 2011; Vaquero \& Trigo 2014; Lockwood et al. 2014b). This series, however, contains a large number of generic no-spot statements (i.e. that no spots were seen on the Sun during long periods), which should be treated with caution (Kovaltsov et al. 2004; Vaquero 2007; Clette et al. 2014; Zolotova \& Ponyavin 2015; Vaquero et al. 2015a, see also Sect. 2.3). Figure 1 also shows two recent estimates of the annual GSN by Vaquero et al. (2015a), who treat generic no-sunspot records in the HS98 catalogue in a conservative way. The sunspot numbers were estimated using the activevs. -inactive day statistics (see Sect. 2.1, with full details in Vaquero et al. 2015a). All these results fall close to each other and imply very low sunspot activity during the MM. On the contrary, Zolotova \& Ponyavin (2015), henceforth called ZP15, argue for higher sunspot activity in the MM (the red dotted curve

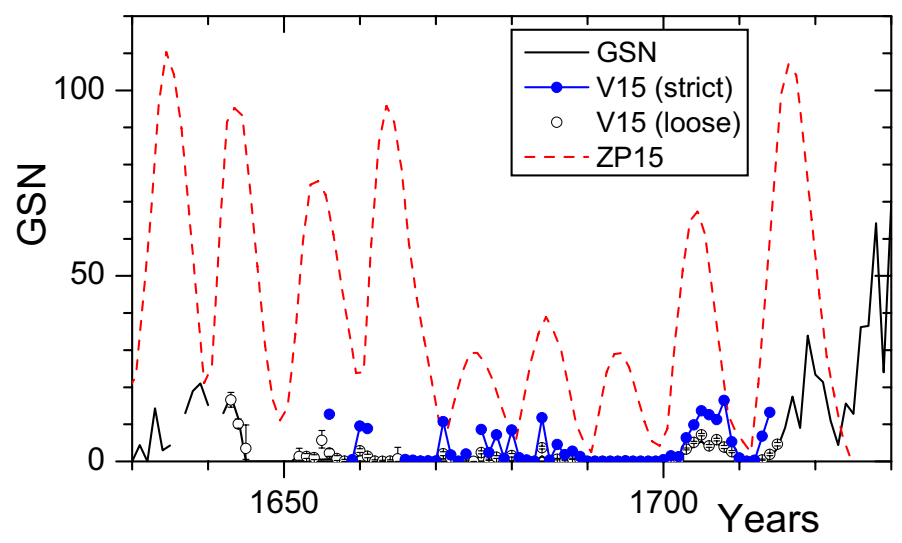

Fig. 1. Annual group sunspot numbers during and around the Maunder minimum, according to Hoyt \& Schatten (1998) - GSN, Zolotova \& Ponyavin (2015) - ZP15, and loose and strictly conservative models from Vaquero et al. (2015a) (see Sect. 2.1), as denoted in the legend.

in Fig. 1 is taken from Fig. 13 of ZP15), with the sunspot cycles not being smaller than a GSN of 30, and even reaching 90-100 during the core MM.

For subsequent analysis we consider two scenarios of solar activity that reflect opposing views on the level of solar activity around the MM before 1749: 1) L-scenario of low activity during the MM, as based on the conventional GSN (Hoyt \& Schatten 1998 ) with recent corrections implemented (see Lockwood et al. 2014b, for details) - and which appear as the black curve in Fig. 1; 2) H-scenario of high activity during the MM, based on GSN as proposed by ZP15 shown as the red dotted curve in Fig. 1). This last scenario also qualitatively represents other suggestions of high activity (e.g., Nagovitsyn 1997; Ogurtsov et al. 2003; Volobuev 2004). After 1749, both scenarios are extended by the international sunspot number ${ }^{1}$. We use annual values throughout the paper unless another time resolution is explicitly mentioned.

\subsection{Fraction of active days}

High solar cycles imply that $\sim 100 \%$ of days are active with sunspots being seen on the Sun almost every day during such cycles, with the exception of a few years around cycle minima (Kovaltsov et al. 2004; Vaquero et al. 2012, 2014). If sunspot activity was high during the MM, as proposed by the H-scenario, the Sun must have been displaying sunspots almost every day. However, this clearly contradicts the data, since the reported sunspot days, including those reported by active observers, cover only a small fraction of the year, even around the proposed cycle maxima (see Fig. 2 in Vaquero et al. 2015a). Thus, either one has to assume a severe selection bias for observers reporting only a few sunspot days per year when spots were present all the time, or to accept that indeed spots were rare.

During periods of weak solar activity, the percentage of spotless days is a very sensitive indicator of activity level (Harvey \& White 1999; Kovaltsov et al. 2004; Vaquero \& Trigo 2014), and much more precise than the sunspot counts. However, this quantity tends towards zero (e.g. almost all days are active) when the average sunspot number exceeds 20 (Vaquero et al. 2015a). Vaquero et al. (2015a) consider several statistically conservative models to assess the sunspot number during the MM from the active day fraction. The "loose" model ignores all generic no-spot

http://sidc.oma.be/silso/datafiles 
statements and accepts only explicit no-spot records with exact date and explicit statements of no spots on the Sun, while the "strict" model considers only such explicit statements as in the "loose" model, but made by at least two independent observers for the spotless days. In this way, the possibility of omitting spots is greatly reduced since the two observers would have to omit the same spot independently. The strict model can be considered as the most generous upper bound to sunspot activity during the MM. However, it most likely exaggerates the activity by over-suppressing records reporting no spots on the Sun. These models are shown in Fig. 1. One can see that these estimates yield sunspot numbers that do not exceed 5 (15) for the "loose" ("strict") model during the MM.

\subsection{Occidental telescopic sunspot observations: historical perspective}

The use of the telescope for astronomical observations quickly became widespread after 1609 . We know that in the second half of the 17th century there were telescopes with sufficient quality and size to see even small spots. Astronomers of that era also used other devices in their routine observations, such as mural quadrants or meridian lines (Heilborn 1999). However, as proposed by ZP15, the quality of the sunspot data for that period might be compromised by non-scientific biases.

\subsubsection{Dominant world view}

Recently, ZP15 have suggested that 17 th century scientists might have been influenced by the "dominant worldview of the seventeenth century that spots (Sun's planets) are shadows from a transit of unknown celestial bodies", and that "an object on the solar surface with an irregular shape or consisting of a set of small spots could have been omitted in a textual report because it was impossible to recognize that this object is a celestial body". This would suggest that professional astronomers of the 17th century, even if technically capable of observing spots, might have distorted the actual records for politically or religiously motivated, nonscientific reasons. This was the key argument for ZP15 to propose high solar activity during the MM. Below we argue that, on the contrary, scientists of the 17 th century were reporting sunspots quite objectively.

Sunspots: planets or solar features? In the first decades of the 17 th century there was a controversy about the location of sunspots: either on the Sun (like clouds) or orbiting at a distance (like a planet). However, already Scheiner and Hevelius plotted non-circular plots and showed the perspective foreshortening of spots near the limb. In his Accuratior Disquisito, Christoph Scheiner (1612) wrote pseudonymously as "Appelles waiting behind the picture" and detailed the appearance of spots of irregular and variable shape, and finally concluded (Galileo \& Scheiner 2010):

They are not to be admitted among the number of stars, because they are of an irregular shape, because they change their shape, because they [...] should already have returned several times, contrary to what has happened, because spots frequently arise in the middle of the Sun that at ingress escaped sharp eyes, because sometimes some disappear before having finished their course.

Even though Scheiner up until this point had believed that sunspots were bodies or other entities just outside the Sun, he did note all their properties very objectively. Later, Scheiner (1630) concluded in his comprehensive book on sunspots, "Maculæ non sunt extra solem" (spots are not outside the Sun, p. 455ff.) and even "Nuclei Macularum sunt profundi" (the cores of sunspots are deep, p. 506). On the contrary, Smogulecz \& Schönberger (1626) who were colleagues of Scheiner in Ingolstadt and Freiburg-im-Breisgau, respectively, called the spots "stellaæ solares" (solar stars) with the sense of moons. Some authors, especially anti-Copernican astronomers, such as Antonius Maria Schyrleus of Rheita (1604-1660) (see Gómez \& Vaquero 2015) and Charles Malapert (1581-1630), followed the planetary model. On the other hand, Galileo had geometrically demonstrated (using the measured apparent velocities of crossing the solar disc) that spots are located on the solar surface. In fact, the changes in the trajectory of sunspots on the solar surface were an important element of discussion in the context of heliocentrism (Smith 1985; Hutchison 1990; Topper 1999).

It was clear already at that time that sunspots were not planets, due to their form, colour, shape of the spots near the limb and their occasional disappearance in the middle of the disk. A nice example is given in a letter to William Gascoigne (1612-1644), which William Crabtree wrote on 7 August 1640 (Aug. 17 greg.) (Chapman 2004), as published by Derham \& Crabtrie (1711):

I have often observed these Spots; yet from all my Observations cannot find one Argument to prove them other than fading Bodies. But that they are no Stars, but unconstant (in regard of their Generation) and irregular Excrescences arising out of, or proceeding from the Sun's Body, many things seem to me to make it more than probable.

Although some astronomers in the mid-17th century still believed that sunspots were small planets orbiting the Sun, the common paradigm among astronomers of that time was "that spots were current material features on the very surface of the Sun" (Brody 2002, p. 78). Therefore, observers of sunspots during the MM, in particular professional astronomers, did not adhere to the "dominant worldview" of the planetary nature of sunspots and hence were not strongly influenced by it, contrary to the claim of ZP15.

Galileo's trial. The problem in the trial of Galileo was not the Copernican system, but the claim that astronomical hypotheses can be validated or invalidated (an absurd presumption for many people of the early 17 th century) leading to a potential claim of reinterpreting the Bible (Schröder 2002). At that time the planetary system was considered a mathematical tool for computing the motion of planets as precisely as possible, and was not a subject to be proved. This subtle difference was an important issue during the first half of the 17 th century to comply with the requirements of the Catholic Church. While an entire discussion of the various misconceptions about the Galileo trial is beyond the scope of this paper, there are many indications that the nature and origin of celestial phenomena were discussed by scholars of the 17th century, rather than being discounted by a standard world view. We are not aware of any evidence that writing about sunspots was prohibited or generally disliked by the majority of observers in any document.

Shape of sunspots. ZP15 presented a hand-picked selection of drawings to support their statement that "there was a tendency to draw sunspots as objects of a circularized form", but there are plenty of other drawings from the same time showing sunspots of irregular shape and sunspot groups with complex structures. Here we show only a few examples of many. 


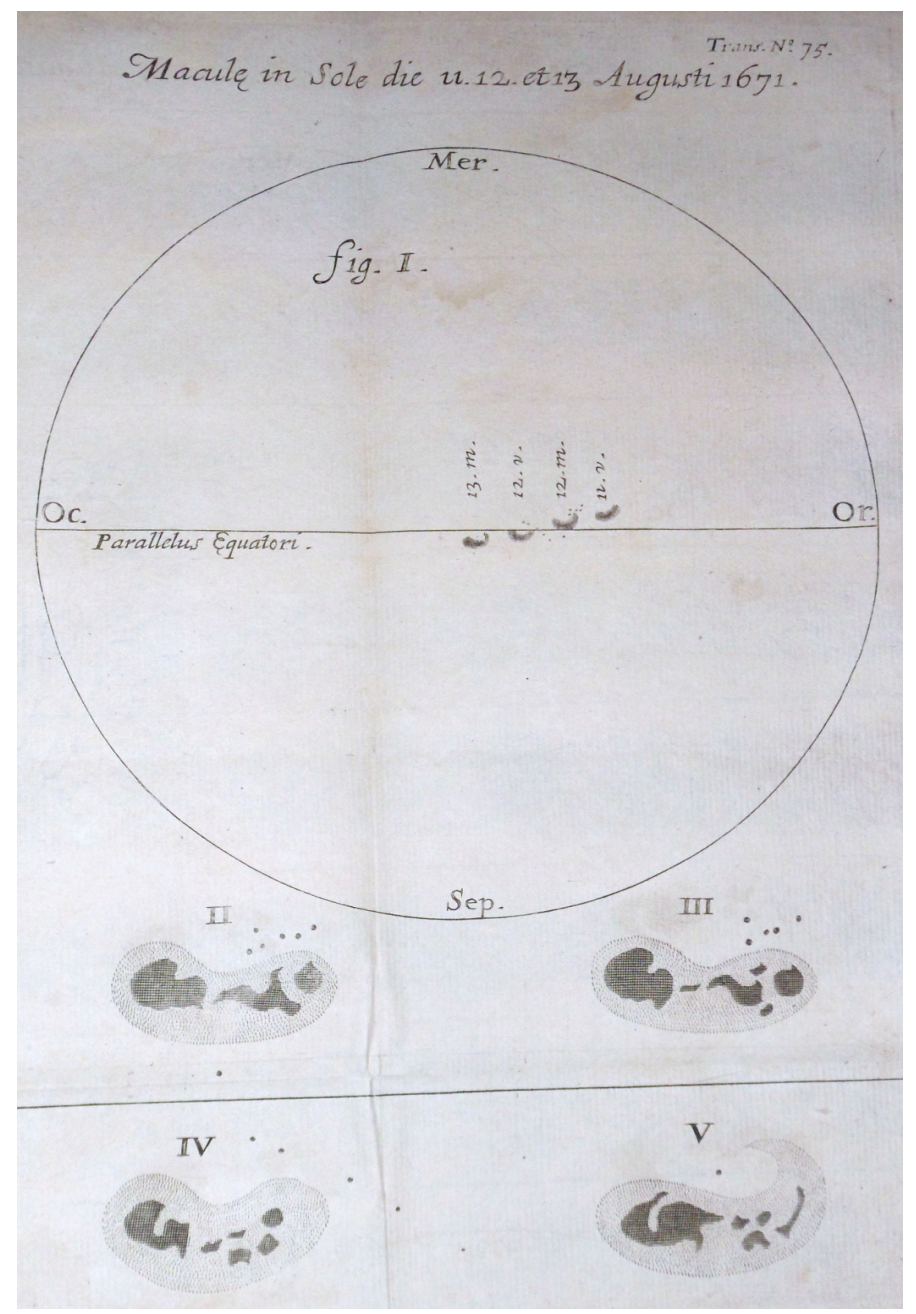

Fig. 2. Drawing of a sunspot group observed in August 1671, as published in number 75 of the Philosophical Transactions, corresponding to August 14, 1671.

Figure 2 depicts a sunspot group observed in several observatories in Europe in August 1671. A dominant spot with a complex structure of multiple umbrae within the same penumbra can be observed with a group of small spots surrounding it. Another example (Fig. 3) shows a drawing of a spot by G. D. Cassini in 1671 (Oldenburg 1671c) ${ }^{2}$, which illustrates the complexity, non-circularity and the foreshortening of sunspots very clearly. Finally, Fig. 4 displays a sunspot observed by J. Cassini and Maraldi from Montpellier (Mar.-29-1701). There is a small sunspot group (labeled as A) more or less in the middle of the solar disc that is magnified in the bottom left corner. This exhibits a complex structure and a legend that reads "Shape of the Spot observed with a large telescope". These drawings are not limited to "circularized forms", and such instances are numerous.

It is important to note that observers who made drawings actually retained the perspective foreshortening of spots near the solar limb. Galilei (1613), Scheiner (1630), Hevelius (1647), G. D. Cassini in 1671 (Oldenburg 1671c), Cassini (1730, observation of 1684), P. De La Hire (1720, observation of 1703), and Derham (1703) all drew slim, non-circular spots near the edge

\footnotetext{
2 Henry Oldenburg was Secretary of the Royal Society and compiled findings from letters of other scientists in the Philosophical Transactions in his own words. We therefore cite his name although it is not given for the actual article.
}

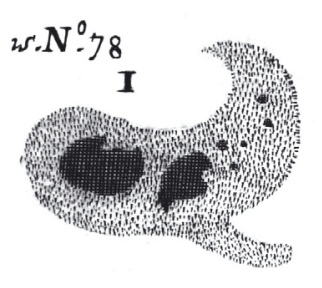

Tab.,
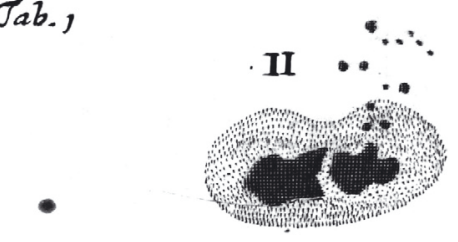

III
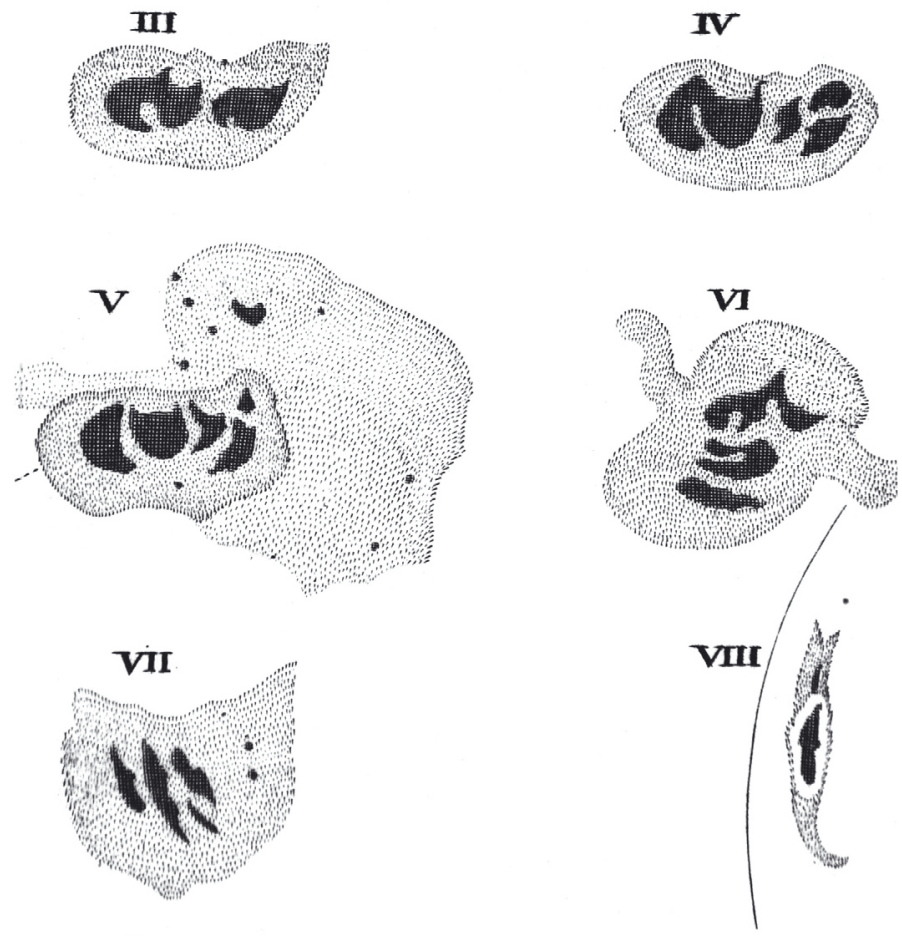

Fig. 3. Sunspot drawings by G. D. Cassini in 1671 (Oldenburg 1671c).

of the Sun. It was clear to them that these objects could not be spheres. They were not shadows either since that would require an additional light source similar to the Sun which is not observed. A note by G. D. Cassini of 1684 says (Cassini 1730):

This penumbra is getting rounder when the spot approaches the center, as it is always happening, this is an indication that this penumbra is flat, and that it looks narrow only because it is presenting itself in an oblique manner, as is the surface of the Sun towards the limb, on which it has to lie.

While G. D. Cassini was an opponent of Copernicus and Newton (Habashi 2007), and in fact discovered a number of Saturn's satellites, he did accept that sunspots appear on the solar surface and did not alter their appearance to make them circular.

Thus, the idea suggested by ZP15 of the strong influence of theological or philosophical ideas about the perfection of the celestial bodies (especially the Sun) on professional astronomers in the late 17th century is not supported by our actual knowledge of solar observations and scientific believes during that time. Once telescopes came into use, the evidence shows that the nature of sunspots were thoroughly discussed using the best available technology at the time leading to a variety of opinions. We further conclude that sunspots were not omitted deliberately from observing records for religious, philosophical or political reasons during the MM. The observational coverage was just incomplete and somewhat vague. Moreover, many existing pieces 


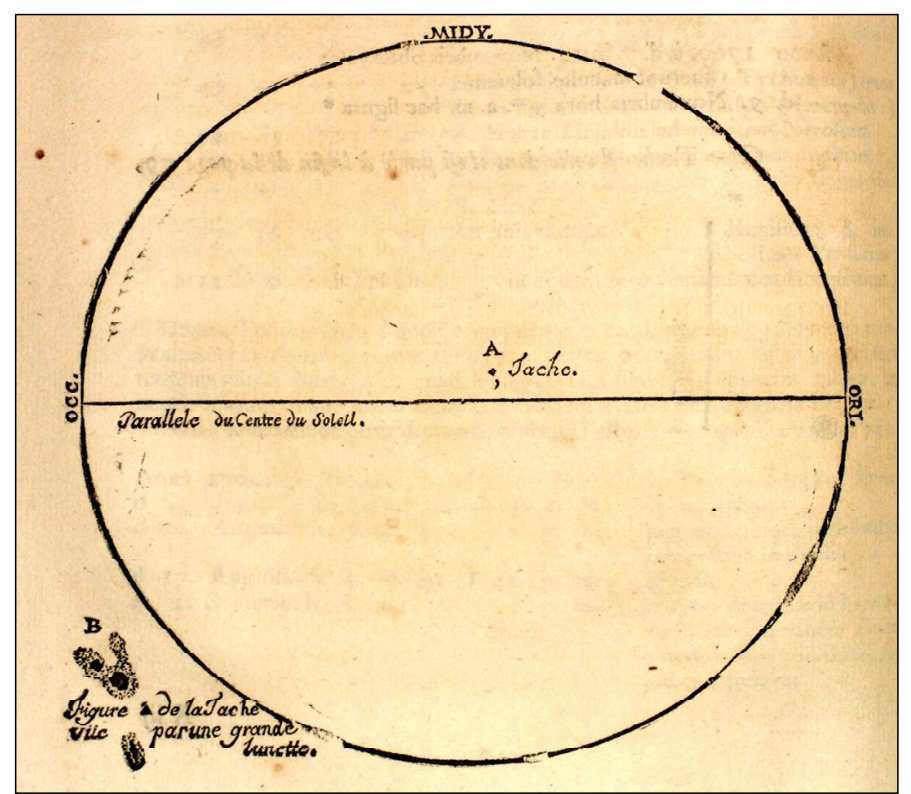

Fig. 4. Sunspot observed by J. Cassini and Maraldi from Montpellier (Mar.-29-1701). Reproduced from page 78 of the Histoire de L'Académie Royale des Sciences (Année MDCCI).

of evidence imply that spots of different shapes were recorded, contrary to the claim of ZP15.

\subsubsection{The very low activity during 1660-1671}

The years 1660-1671 indicate a period of very low activity in the HS98 database, but this is mostly based on generic statements of the absence of sunspots. For example, based on a report by G. D. Cassini, a sunspot observed in 1671 (Oldenburg 1671b) was described in detail, and it was noted that

"it is now about twenty years since, that Astronomers have not seen any considerable spots in the Sun, though before that time $[. .$.$] they have from time to time ob-$ served them. The Sun appeared all that while with an entire brightness".

The last sentence implies that the Sun was also void of any other dark features, even if they would not have been reported in terms of sunspots. There is also a footnote saying that, indeed, some spots were witnessed in 1660 and 1661, so the 20 years mentioned were exaggerated. The Journal also states (Oldenburg 1671a) that "as far as we can learn, the last observation in England of any Solar Spots, was made by our Noble Philosopher Mr. Boyl" on Apr. 27 (May 7 greg.) 1660 and May 25 (Jun. 4 greg.). He described a "very dark spot almost of quadrangular form". Moreover, one of the spots was described as oval, while another was oblong and curved. This statement contradicts the assumption of ZP15 that the majority of non-circular spots were omitted, especially in relation to the tone of surprise with which the article was written, and that spots were seen at all. If there were a number of non-circular spots during this ten-year period (allegedly not reported), there would have been no reason to "celebrate" yet another non-circular spot in 1671 .

In another example, Spörer (1889), p. 315, cited a note by Weigel from Jena in 1665 , which can be translated as

Many diligent observers of the skies have wondered here that for such a long time no spots were noticeable on the
Sun. And we need to admit here in Jena that, despite having tried in many ways, setting up large and small spotting scopes pointed to the Sun, we have not found such phenomena for a considerable amount of time. (translation by AR).

Since the notes on the absence of spots come from various countries and from Catholic, Protestant and Anglican people, we do not believe there was any widespread religious attitude to "suppress" spots to save the purity of the Sun.

The only positive sunspot report between 1660 and 1671 in the HS98 database is the one by Kircher in 1667 . This data point comes from a note (Frick 1681, p. 49) stating that

the late Christoff Weickman, who was experienced in optics and made a number of excellent telescopes, watched the Sun at various times hoping to see the like [sunspots] on the Sun, but could never get a glimpse of them [...] So Mr Weickman wrote to Father Kircher and uncovered him that he could not see such things on the Sun, does not know why this is or where the mistake could be. Father Kircher answered from Rome on 2 September 1667 that it happens very rarely that one could see the Sun as such; he had not seen it in such a manner more than once, namely Anno 1636.

One can see that the date of the letter in 1667 was mistakenly considered as the observation date. Instead the report clearly indicates that no sunspots were seen at all by Weickman in the 1660s. The sunspot observation by Kircher in 1667 is erroneous and needs to be removed from the HS98 database. Then no record of sunspots exists in the 1660s. We note that this false report was used by ZP15 to evaluate the sunspot cycle maximum around that date.

\subsection{Generic statements and gaps in the HS98 database}

The database of HS98 forms a basis for many studies of sunspot records during the period under investigation. In particular, ZP15 based their arguments on this database without referring to the original records. However, the database contains several not obvious features that can be easily misinterpreted if not considered properly. Here we discuss these features that are directly related to the evaluation of sunspot activity in the 17 th century.

In particular, many no-spot records were related to astrometric observations of the Sun such as the solar meridian altitude or the apparent solar diameter (Vaquero \& Gallego 2014). For example, Manfredi (1736) listed more than 4200 solar meridian observations made by several scientists during the period 1655 1736, using the gigantic camera obscura installed on the floor of the Basilica of San Petronio in Bologna. These observations were not focused on sunspots and did not include any mention of spots. However, HS98 treated all these reports as observations of the absence of sunspot groups, which, of course, was incorrect.

HS98 database also contains gaps in the observing records of Marius and Riccioli, which occur exactly during days when other observers reported spots. This was interpreted by ZP15 as indications that they deliberately stopped reporting to hide sunspots: "it is noteworthy that when the Sun became active, Marius and Riccioli immediately stopped observations". We note that this interpretation is erroneous and based on ignorance of the detail of the HS98 database as explained below.

The original statement by Marius from Apr 16 (=Apr 26 greg.) 1619, on which this series is based, is

While I did not find as many spots in the disk of the Sun over the past one-and-a-half years, often not even a single 
spot, which was never seen in the year before, I noted in my observing diary: Mirum mihi videtur, adeo raras vel sæpius nullas maculas in disco solis deprehendi, quod ante hâc nunque est observatum

which is a repetition of what he said before in Latin. Marius clearly states that the number of sunspot was not exactly zero, but very low. HS98 have used this statement to approximate the activity by zeros in their database more precisely by filling all dates in the 1.5-yr interval with zeros except for the periods when other observers did see spots. The existence of gaps is by no means based on the actual reports by Marius, but is an artefact of the way HS98 have interpreted the original comment.

The same reason holds for the gaps in sunspots reported by Riccioli (1653), p. 96, whose data (zeros) in the HS98 database are based on the statement that

.... in the year 1618 when a comet and tail shone, no spots were observed, said Argolus in Pandosion Sphæricum chap. 44.

The original statement by Argolus (1644), p. 213, states: "Anno 1618 tempore quo Trabs, et Cometa affulsit nulla visa est". Apart from the fact that it was not Riccioli himself who made the observations, this again led to filling all days in 1618 with zeros (in the HS98 database), except for the days when other observers saw spots.

The method of filling the HS98 database for many months and even years with zeros is based on generic verbal reports on the absence of spots for long periods also in the cases of Picard, G. D. Cassini, Dechales, Maraldi, Siverus and others (see, e.g, Vaquero et al. 2011, 2015a). HS98 must have filled those periods in the sense of probably very low activity, but they are not meant to provide exact timings of observations, as ZP15 interpreted them. The appearance of gaps in zero records when other observers reported spots is not an indication of withholding spots in observing reports but rather a simple technical way of avoiding conflicting data in the HS98 database. ZP15 mistook the entries in the HS98 database for actual observing dates and interpreted them incorrectly.

While assuming a large number of days without spots is a significant underestimation of solar activity, on the one hand, as demonstrated by Vaquero et al. (2015a) and as pointed out by ZP15 as well, the assumption that observers deliberately stopped reporting is, on the other hand, not supported by any original text and remains ungrounded speculation.

The observations by Hevelius of 1653-1684, as recovered by Hoyt \& Schatten (1995), should also be scrutinized with regard to a possible omission of spots. Citing the former reference, ZP15 even claim that "Hevelius quite consciously did not record sunspots", while the original statement claims that "Hevelius occasionally missed sunspots but usually was a reliable observer". Actually, out of 24 groups that could have been detected by Hevelius taking into consideration his observing days, he saw 20 (Hoyt \& Schatten 1995). He never reported the absence of sunspots when others saw them. The four occasions are simply not accompanied by any statement about presence or absence of spots. This can be interpreted as the sunspot notes were just remarks on his solar elevation measurements (Hevelius 1679, part 3). These, however, were made with a quadrans azimutalis which had no telescope, since Hevelius refused to switch to a telescope at some point, perhaps because he did not want to spoil his time series of measurements (Habashi 2007). He therefore could not see sunspots at all with his device and had to use an additional instrument to observe them, and it is probable that he did not do so on each day he measured the solar elevation, hence why he left so many days with neither positive nor negative information on sunspots. We have to treat these as non-observations.

\subsection{Methodological errors of ZP15}

The original work by ZP15 unfortunately contains a number of methodological errors which eventually led them to an extreme conclusion that sunspot activity during the MM was at a moderate to high level. In particular, ZP15 sometimes incorrectly interpreted published records. Moreover they used the original uncorrected record of HS98, while numerous corrections have been made during the past 17 years (e.g. Vaquero et al. 2011; Vaquero \& Trigo 2014; Carrasco et al. 2015). Here we discuss in detail some of the errors in ZP15, as examples of erroneous interpretation of historical data.

\subsubsection{Sunspot drawings vs. textual notes}

According to ZP15 "sunspot drawings provide a significantly larger number of sunspots, compared to textual or tabular sources". This is trivial considering the tabular sources are often related to astrometric observations of the Sun, such as solar meridian altitude or the apparent solar diameter (Vaquero \& Gallego 2014). However, if one considers only those tabular sources that contain explicit information about the presence or absence of sunspots then drawing sources appear to be consistent with the reliable tabular sources (Kovaltsov et al. 2004; Carrasco et al. 2015).

The main assumption in ZP15 is that sunspots were omitted, especially in verbal reports, if they were not round and did not resemble a planet. The only direct example of that is given by ZP15, with a reference to Vaquero \& Vázquez (2009), where Harriot drew three sunspots on Dec. 8 (Dec. 18 greg.) 1610 but wrote that the Sun was "clear". However, the assumption by ZP15 was based on an incorrect interpretation of the original texts. The actual statement of Harriot (1613) is

The altitude of the sonne being 7 or 8 degrees. It being a frost and a mist. I saw the sonne in this manner [drawing]. I saw it twise or thrise. Once with the right ey and other time with the left. In the space of a minute time, after the sonne was to cleare.

As indicated by the observing times and numerous other statements about a "well tempered" Sun in the course of his observations, he mostly made his observations around sunrise or sunset, or with a degree of cloud cover to be able to look through the telescope. The statement that "the sonne was to cleare" refers to the fact that the Sun became too bright after a few minutes of observing. In this context, "cleare" means "bright" and not clear or spotless. Therefore, this example was incorrectly taken by ZP15 as an illustrative case of the discrepancy between textual and drawn sources.

As another example, we compared the textual records of Smogulecz \& Schönberger (1626), who had conservative views on sunspots (see Sect. 2.2.1), with the drawings made by Scheiner in Rome for the same period of 1625 . We found that that Smogulecz and Schönberger omitted a number of spots from their drawings, but mentioned all the spots they saw in their text (calling them "stellæ"), in accordance with Scheiner. This contradicts the assumption of ZP15 that verbal reports are subject to withholding spots. Table 1 lists the numbers of 
Table 1. Comparison of the number of spots listed in the verbal reports versus the number of spots in the drawings by Smogulecz \& Schönberger (1626) in 1625.

\begin{tabular}{lrr|lrr}
\hline \hline Date & Text & Drawn & Date & Text & Drawn \\
\hline 1625 Jan. 14 & 1 & 1 & 1625 Aug. 22 & 2 & 1 \\
1625 Jan. 15 & 1 & 1 & 1625 Aug. 23 & 2 & 1 \\
1625 Jan. 16 & 4 & 1 & 1625 Aug. 27 & 6 & 1 \\
1625 Jan. 17 & 8 & 1 & 1625 Aug. 28 & 10 & 1 \\
1625 Jan. 18 & 2 & 1 & 1625 Aug. 31 & 7 & 1 \\
1625 Jan. 19 & 4 & 1 & 1625 Sep. 01 & 6 & 1 \\
1625 Jan. 20 & 2 & 1 & 1625 Sep. 05 & 8 & 4 \\
1625 Feb. 12 & 8 & 1 & 1625 Sep. 07 & 6 & 3 \\
1625 Feb. 16 & 10 & 1 & 1625 Sep. 08 & 6 & 3 \\
1625 Feb. 17 & 11 & 1 & 1625 Sep. 11 & 5 & 3 \\
1625 Feb. 18 & 10 & 1 & 1625 Sep. 12 & 4 & 3 \\
1625 Feb. 21 & 4 & 1 & 1625 Sep. 13 & 2 & 2 \\
1625 Jun. 01 & 9 & 1 & 1625 Oct. 05 & 9 & 8 \\
1625 Jun. 04 & 3 & 1 & 1625 Oct. 06 & 2 & 1 \\
1625 Jun. 05 & 3 & 1 & 1625 Oct. 09 & 4 & 4 \\
1625 Jun. 06 & 2 & 1 & 1625 Oct. 10 & 7 & 8 \\
1625 Jun. 07 & 3 & 1 & 1625 Oct. 11 & 9 & 9 \\
1625 Jun. 09 & 2 & 1 & 1625 Oct. 13 & 2 & 1 \\
1625 Aug. 08 & 6 & 1 & 1625 Oct. 14 & 2 & 1 \\
1625 Aug. 09 & 4 & 1 & 1625 Oct. 15 & 3 & 1 \\
1625 Aug. 10 & 2 & 1 & 1625 Oct. 25 & 1 & 1 \\
1625 Aug. 12 & 4 & 2 & 1625 Oct. 26 & 1 & 1 \\
1625 Aug. 13 & 3 & 2 & 1625 Oct. 27 & 1 & 1 \\
1625 Aug. 14 & 3 & 2 & 1625 Oct. 28 & 1 & 1 \\
1626 Aug. 15 & 4 & 2 & 1625 Oct. 29 & 1 & 1 \\
1625 Aug. 17 & 2 & 2 & 1625 Oct. 31 & 1 & 1 \\
1625 Aug. 18 & 4 & 2 & 1625 Nov. 01 & 1 & 1 \\
1625 Aug. 19 & 2 & 1 & & & \\
\hline
\end{tabular}

spots mentioned in their text versus those drawn in the figures. (We note that the values are also incorrectly used in HS98.) Smogulecz \& Schönberger (1626) selected certain spots that were visible long enough to measure the obliquity of the Sun's axis with the respect to the ecliptic and plotted them schematically as circles since they were not particularly interested in their shape.

\subsubsection{Relation between maximum number of sunspot groups and sunspot number}

ZP15 proposed a new method to assess the amplitude of the solar cycle during the MM. As the amplitude of a sunspot cycle, $A_{G}^{*}$, they used the maximum daily number of sunspot groups $G^{*}$ during the cycle, so that $A_{G}^{*}=12.08 \times G^{*}$, where the coefficient 12.08 is a scaling between the average number of sunspot groups and the sunspot number (Hoyt \& Schatten 1998). We note that using the maximum daily value of $G^{*}$ instead of the average value $G$ leads to a large overestimate of the sunspot cycle amplitude, particularly during the MM. We analyzed the HS98 database for the period 1886-1945, when sunspot cycles were not very high, to compare the annually averaged group sunspot numbers $R_{G}$ and the annual values of $A_{G}^{*}$ obtained using the annual maxima of the daily sunspot group numbers $G^{*}$. Figure 5a shows a scatter plot of the annual values of $R_{G}$ and $G^{*}$ (dots), while the dashed line gives an estimate of the $A_{G}^{*}$ based on $G^{*}$, following the method of ZP15. One can see that while there is a relation between annual $R_{G}$ and $G^{*}$, the proposed method heavily overestimates annual sunspot activity. Figure $5 \mathrm{~b}$ shows the overestimate factor $Y=A_{G}^{*} / R_{G}$ of the sunspot numbers as a function of $G^{*}$. While the factor $Y$ is $2-3$ for very active years

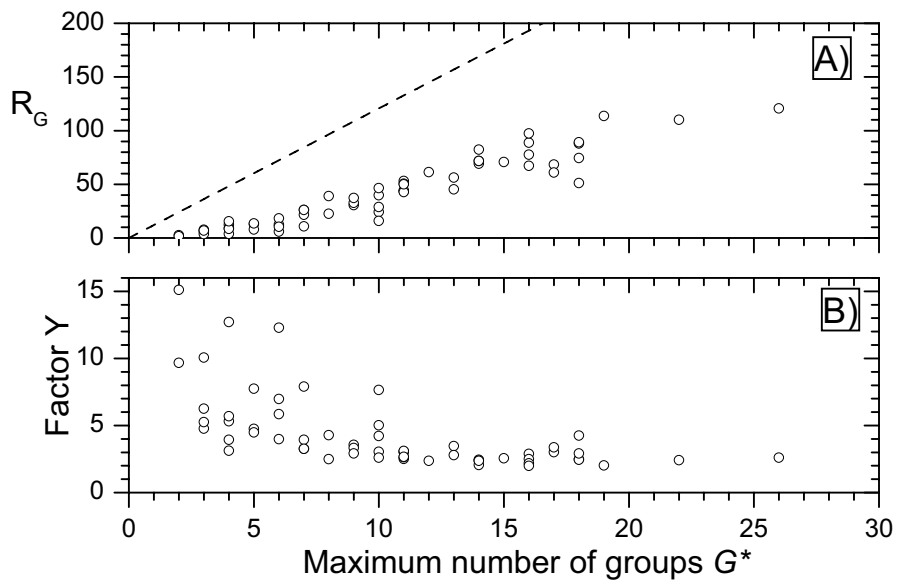

Fig. 5. Illustration of the incorrectness of the method used by ZP15 to assess the group sunspot number $R_{G}$ during the MM. Panel A) annual values of $R_{G}$ as a function of the maximum daily number of sunspot groups $G^{*}$ for the same year in the HS98 catalogue for the period of 1886-1945; the dashed line is the dependence of $A_{G}^{*}=12.08 \times G^{*}$ used by ZP15. Panel B) the overestimate factor $Y$ of the GSN by the ZP15 method $Y=A_{G}^{*} / R_{G}$.

with $G^{*}>15$, the overestimate can reach an order of magnitude for years with weaker activity, such as during the MM. When applied to the sunspot cycle amplitude, the error becomes even more severe. Thus, by taking the cycle maxima of the daily number of sunspot groups instead of their annual means, ZP15 systematically overestimated the sunspot numbers during the MM by a factor of 5-15.

The number of sunspot groups in 1642. ZP15 proposed that the solar cycle just before the MM was high (sunspot number $\approx 100$ ) which is based on a report of 8 sunspot groups observed by Antonius Maria Schyrleus of Rheita in February 1642 as presented in the HS98 database. However, as shown by Gómez \& Vaquero (2015), this record is erroneous in the HS98 database because it is based on an incorrect translation of the original Latin records, which say that one group (or a few) was observed for eight days in June 1642 instead of eight groups in February 1642. Accordingly, the maximum daily number of sunspot groups reported for that cycle $G^{*}$ was five, not eight, reducing the cycle amplitude claimed by ZP15 (see Sect. 2.4.2) by about $40 \%$.

The number of sunspot groups in 1652. The original HS98 record contains five sunspot groups for the day of Apr. 01 1652, referring to observations by Johannes Hevelius. Accordingly, this value (the highest daily $G^{*}$ for the decade) was adopted by ZP15 leading to the high proposed sunspot cycle during the 1650s. However, as discussed by Vaquero \& Trigo (2014) in great detail, this value of five sunspot groups is an erroneous interpretation made by HS98 with reference to Wolf (1856), of the original Latin text by Hevelius, which says that there were five spots in two distinct groups on the Sun. Accordingly, the correct value of $G^{*}$ for that day should be two not five.

The number of sunspot groups in 1705. A high sunspot number of above 70 was proposed by ZP15 for the year 1705 based on six sunspot groups reported by J. Plantade from Montpellier (the correction factor for this observer is 1.107 according to HS98) for the day Feb. 13, 1705. This observer was quite active with regular observations during that period, with 44 known sightings for the year 1705. For example, J. Plantade reported two, three, six, and one groups, respectively, for the days of Feb. 11 through Feb. 14. His reports also mention the explicit 
absence of spots from the Sun after the group he had followed passed beyond the limb. However, he did not make any reports during long spotless periods, and wrote notes again when a new sunspot group appeared. The average number of sunspot groups per day reported by J. Plantade for 1705 was 1.22 , which is a factor of five lower than that adopted by ZP15 who only took the largest daily value (see Sect. 2.4.2). If one calculates the group sunspot number from the dataset of J. Plantade records for 1705 in the classical way, one obtains a value of $R_{G}=16.3=$ $1.22 \times 12.08 \times 1.107$.

\subsection{Butterfly diagram}

According to sunspot drawings during some periods of the MM, a hint of the butterfly diagram was identified, particularly towards the end of the MM after 1670 (Ribes \& Nesme-Ribes 1993; Soon \& Yaskell 2003; Casas et al. 2006). However, the latitudinal extent of the butterfly wings was quite narrow, being within $15^{\circ}$ for the core MM (1645-1700) and $20^{\circ}$ for the period around 1705 , while cycles before and after the MM had a latitudinal extent of $30^{\circ}$ or greater. This suggests that the sunspot occurrence during the MM was limited to a more narrow band than outside the MM. Here we compare the statistics of the latitudinal extent of the butterfly diagram wings for solar cycles \#0 through 22 (cycles 5 and 6 are missing). The cycles \#0 through 4 were covered by digitized drawings made by Staudacher for the period 1749-1792 (Arlt 2008), cycles \#7 through 10 (1825-1867) were covered by digitized drawings made by Samuel Heinrich Schwabe (Arlt et al. 2013), cycle \#8 by drawings of Gustav Spörer (Diercke et al. 2015), while the period after 1874 was studied using the Royal Greenwich Observatory (RGO) catalogue. Moreover, a machine-readable version of the sunspot catalogues of the 19th century complied by Carrington, Peters and de la Rue has been released recently (Casas \& Vaquero 2014). For each solar cycle we define the maximum latitude (in absolute values without differentiating north and south) of sunspot occurrence. Since the telescopic instruments were poorer during the MM than nowadays, for consistency we considere only large spots with the projected spot area greater than $100 \mathrm{msd}$ (millionths of the solar disc). The result is shown in Fig. 6 as a function of the cycle maximum (in $R_{G}$ ), which demonstrates that there is a weak dependence for stronger cycles generally having a larger latitudinal span (see, e.g., Vitinsky et al. 1986; Solanki et al. 2008; Jiang et al. 2011b), but the latitudinal extent of the butterfly wing has always been greater than $28^{\circ}$ for the last 250 years. A robust link between the mean/range latitude of sunspot occurrence and cycle strength is related to the dynamo wave in the solar convection zone which has been empirically studied, e.g., by Solanki et al. (2008) or Jiang et al. (2011a). Since the maximum latitudinal extent of sunspots during the $\mathrm{MM}$ was $15^{\circ}$ (during the core MM) or $20^{\circ}$ (around ca. 1705), it suggests a weak toroidal field, causing a narrower latitudinal range of sunspot formation during the MM. This conclusion agrees with the results of more sophisticated analysis by Ivanov \& Miletsky (2011), who found that the latitudinal span of the butterfly diagram during the late part of the MM should be 15$20^{\circ}$, i.e., significantly lower than during normal cycles. One may assume that all the higher latitude spots were deliberately omitted by all observers during the MM but we are not aware of such a bias.

We note that two datasets of sunspot latitudes during the $\mathrm{MM}$ have been recently recovered and translated into machine-readable format (Vaquero et al. 2015b). Using these datasets, a decadal hemispheric asymmetry index has been

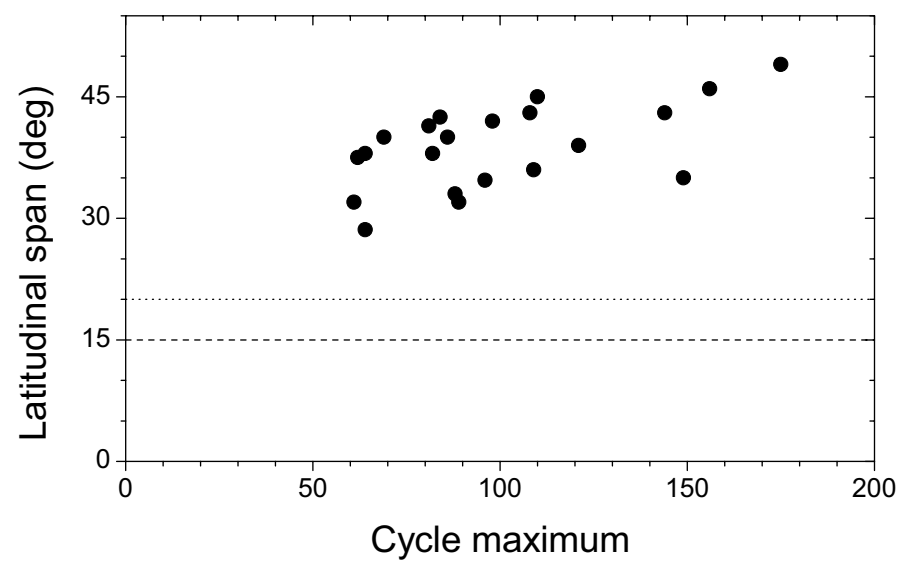

Fig. 6. Maximum latitudinal span of the butterfly diagram as a function of the cycle amplitude in annual $R_{G}$ for solar cycles \# 0-4 and 7-22, accounting only for large spots (with an area greater than $100 \mathrm{msd}$ ). The dashed and dotted lines depict the maximum latitudinal extent of sunspot occurrence during the core (1645-1700) and the entire MM (1645-1712).

calculated confirming a very strong hemispherical asymmetry (sunspots appeared mostly in the southern hemisphere) in the MM, as reported in earlier works (Spörer 1889; Ribes \& Nesme-Ribes 1993; Sokoloff \& Nesme-Ribes 1994). Another moderately asymmetric pattern was observed only at the beginning of the DM (Arlt 2009; Usoskin et al. 2009b). Thus, this indicates that the MM was also a special period with respect to the distribution of sunspot latitudes.

\subsection{East-Asian naked-eye sunspot observations}

East-Asian chronicles reporting observations for about two millennia, by unaided naked eyes, of phenomena that may be interpreted as sunspots have sometimes been used as an argument suggesting high solar activity during the MM (Schove 1983; Nagovitsyn 2001; Ogurtsov et al. 2003; Zolotova \& Ponyavin 2015). Such statements are based on an assumption that sunspots must be large to be observed and that this is possible only at a high level of solar activity. However, as shown below, this is not correct. While such historical records can be useful in a longterm perspective showing qualitatively the presence of several grand minima during the last two millennia (Clark \& Stephenson 1978; Vaquero et al. 2002; Vaquero \& Vázquez 2009) including also the MM, this dataset is not useful for establishing the quantitative level of solar activity over short timespans due to the small number of individual observations and/or the specific meteorological, sociological and historical conditions required for such records (see Chap. 2 in Vaquero \& Vázquez 2009). Moreover, it is very important to indicate that the quality of the historical record of naked-eye sunspot (NES) observations was not uniform through the ages (i.e. during the approximately two milliennia covered by the record). In fact, the quality of such records for the last four centuries was much poorer than that for the $12-15$ th centuries due to a change in the type of historical sources. In particular, data coverage was reduced greatly after 1600 (see Fig. 2.18 in Vaquero \& Vázquez 2009). There are very few NES records during the century between the MM and the DM, representing the social conditions supporting such observations and the maintenance of such records rather than sunspot activity. Therefore, the historical record of NES observations is 


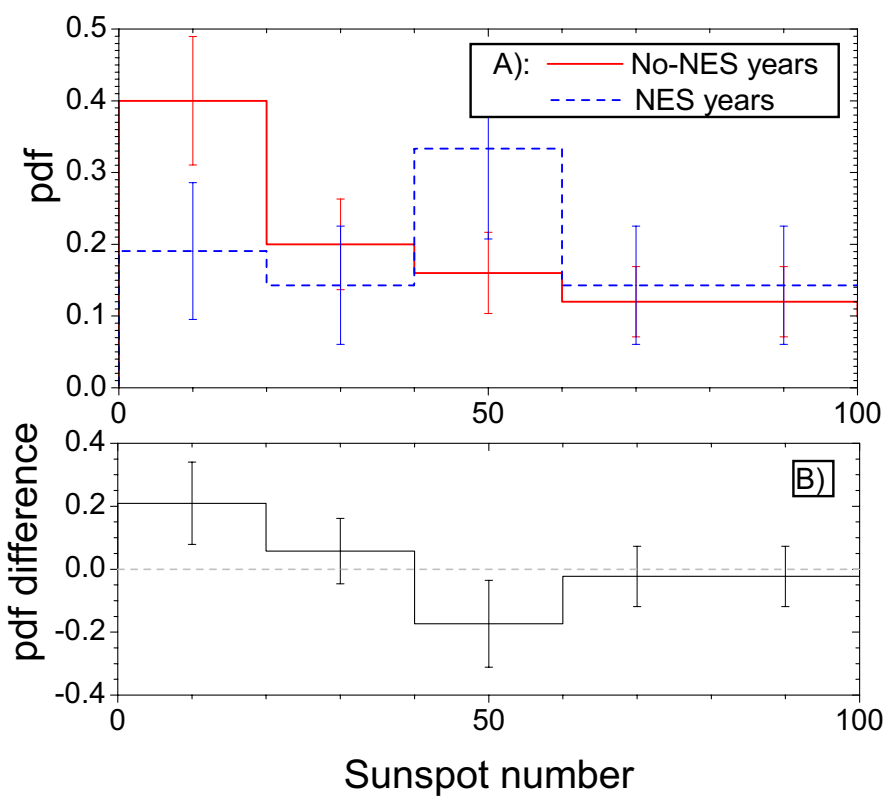

Fig. 7. Probability density function for occurrence of the annual group sunspot numbers for the years 1848-1918. Panel A) the red solid line represents the years (50 years) without naked-eye spot (no-NES) reports, while the blue dotted line represents only the years (21 years) with NES. Panel B) the difference between the no-NES and NES probability density functions. Error bars represent the $1 \sigma$ statistical uncertainties.

not useful to estimate the level of solar activity during recent centuries (Eddy 1983; Mossman 1989; Willis et al. 1996).

\subsubsection{Do NES observations imply high activity?}

It is typical to believe that historical records of NES observations necessarily imply very high levels of solar activity (e.g., Ogurtsov et al. 2003), assuming that observable spots must have a large area exceeding 1900 msd (millionths of the solar disc) with a reference to Wittmann (1978). However, this work does not provide any argumentation for such a value and, as shown below, is not correct.

Here we show that reports of NES observations do not necessarily correspond to high activity or even to big spots. We compared the East-Asian sunspot catalogue by Yau \& Stephenson (1988) for the period 1848-1918 (25 reported naked-eye observations during 21 years) with data from the HS98 catalogue. Figure 7 shows the probability density function (pdf) of the sunspot numbers for the years with and without NES observations. One can see that the probability of NES reports to occur does not depend on actual sunspot number as the blue dotted curve in panel A is almost flat while, intuitively, it should yield higher probability for high sunspot numbers and vanish for small sunspot numbers. Moreover, there is no statistically significant difference in sunspot numbers between the two pdfs. Accordingly, the null hypothesis that the two pdfs belong to the same population cannot be statistically rejected. Obviously, there is no preference to NES observations during the years of high sunspot numbers. The naked-eye reports appear to be distributed randomly, without any relation to actual sunspot activity. Accordingly, the years with unaided naked-eye sunspot reports provide no preference for higher sunspot number.

Next we study the correspondence between the NES reports and actual sunspots during the exact dates of NES observations (allowing for 1 day dating mismatch because of the local time conversion). The data on the sunspot area were taken from the

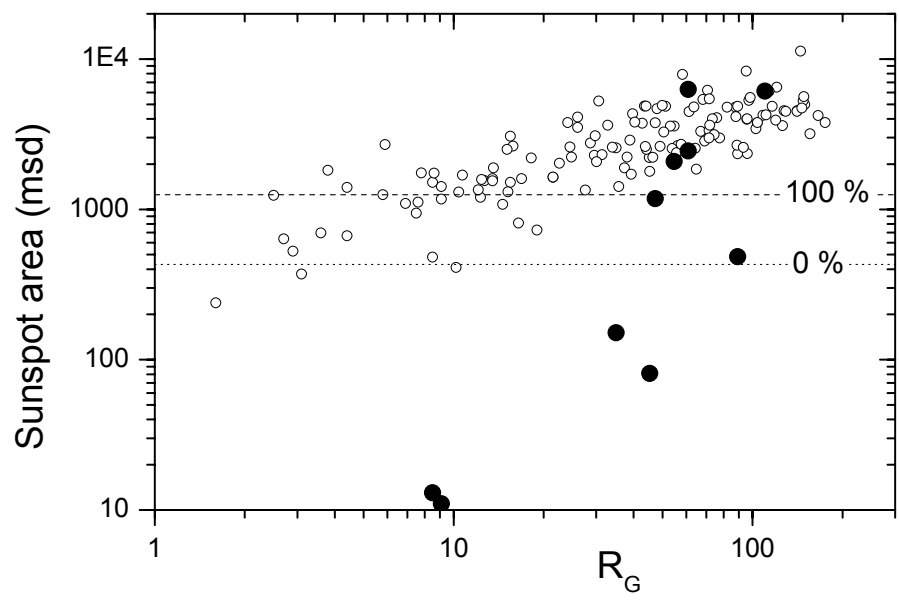

Fig. 8. Open dots depict dependence of the area of the largest sunspot within a year (GSO data) vs. the annual group (1874-1996) or international (1997-2013) sunspot numbers. Big filled dots denote the largest sunspot's area for the days of reported NES observations during the period 1874-1918 (Yau \& Stephenson 1988). The dashed and dotted lines depict the $100 \%$ (all spots above this line are visible) and $0 \%$ (detectability threshold) probability of observing a sunspot of the given area by an unaided eye, according to Schaefer (1993) and Vaquero \& Vázquez (2009).

RGO sunspot group photographic catalogue ${ }^{3}$. Figure 8 shows, as filled circles, the largest observed sunspot area for the days when East-Asian NES observation were reported during the years 1874-1918 (Yau \& Stephenson 1988). Detectability limits of the NES observations (Schaefer 1993; Vaquero \& Vázquez 2009) are shown as dotted (no spots smaller than $\approx 425$ msd can be observed by the unaided eye) and dashed (all spots greater than $\sim 1240$ msd are observable) lines. Half of the reported NESs lie below or at the lower detectability limit and are not visible by a normal unaided human eye, meaning they are likely to be spurious or misidentified records (cf. Willis et al. 1996).

As an example we consider two dates with NES records with the smallest sunspots. A sunspot was reported as being seen by the naked eye on Feb. 15, 1900, when there were no sunspots on the Sun according to RGO, while there was one very small group (11 msd area) on the pervious day of Feb. 14, 1900. Another example of a NES report is for the day of Jan. 30, 1911 when there was a single small group (area $13 \mathrm{msd}$ ) on the Sun (see also Fig. 9 in Yau \& Stephenson 1988). Such small groups cannot be observed by an unaided eye. Moreover, in agreement with the above discussion, even for big spots above the $100 \%$ detectability level, the relation to solar activity is unclear. Open dots in Fig. 8 denote the area of the largest spot observed each year vs. the mean annual sunspot number for years 1874-2013. One can see that the occurrence of a large sunspot detectable by the naked eye does not necessarily correspond to a high annual sunspot number, as it can occur at any level of solar activity from $R_{G}=3$ to 200 .

Another dataset is provided by the naked-eye observations by Samuel Heinrich Schwabe, who recorded telescopic sunspot data in 1825-1867, but also occasionally reported on naked-eye visibilities of sunspot groups (Pavai et al., in prep.). We analyzed the (annual) group sunspot numbers for each event when Schwabe reported a naked-eye visibility, as shown in Fig. 9, in the form of a probability density function versus the annual

http://solarscience.msfc.nasa.gov/greenwch.shtml 


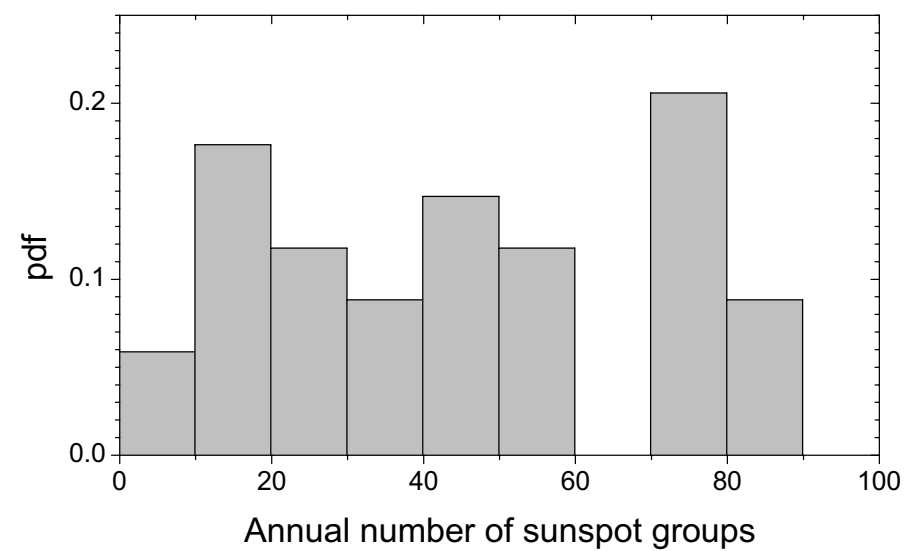

Fig. 9. Probability density function of the occurrence of NES records by $\mathrm{S}$. H. Schwabe as a function of the annual groups sunspot number during the years of Schwabe's observations.

group sunspot number. The NES reports were quite frequent during years with low sunspot activity $(\approx 25 \%$ of NES were reported for the years with $R_{G}$ below 20, some of them even below 10). It is interesting that about $20 \%$ of naked eye observations by Schwabe were reported on days with a single group on the solar disc. We note that Schwabe certainly looked with the naked eye when he saw a big group with a telescope, so the selection may be biased towards larger spots. On the other hand, it is unlikely that he would identified NES only if there were few groups on the Sun. On this basis we do not expect an observational bias towards lower activity periods.

Based on the above, a significant part of the East-Asian NES observational reports are unlikely to be real observations and, even if they were correct, they do not imply a high level of solar activity. This implies that NES reports cannot be used as an index of sunspot activity in a straightforward way (cf. Eddy 1983; Willis et al. 1996; Mossman 1989; Usoskin 2013).

\subsubsection{NES observations around the MM}

According to the well-established catalogue of NESs (Yau \& Stephenson 1988) from oriental chronicles, NESs were observed relatively frequently before the $\mathrm{MM}-16$ years during the period 1611-1645 are marked with NES records. A direct comparison between the NES catalogue and the HS98 database (with the correction by Vaquero et al. 2011) shows that NES records either are confirmed by Eurpean telescopic observations (Malapert, Schenier, Mogling, Gassendi, Hevelius) or fall in data gaps (after removing generic statements from the HS98 database). There are no direct contradictions between the datasets for that period.

However, there are several NES records also during the MM but they are more rare (8 years during 1645-1715), as discussed in detail here. Three NES observations were reported for the years 1647,1648 , and 1650, which appear during a long gap (1646-1651) of telescopic observations where only a generic statement by Hevelius exists. The exact level of sunspot activity during these years is therefore unknown. A NES report dated Apr. 30 (greg.) 1655 falls in a small gap in the HS98 database but there is some activity reported in the previous month of March. The mean annual GSN for the year 1655, estimated in the "loose" model of Vaquero et al. (2015a), is $R_{G}=5.7$.

In the Spring of 1656 a NES was reported, which overlaps with a sunspot group reported by Bose in February. $R_{G}$ is estimated in the "strict" model as 12.7 (Vaquero et al. 2015a). There are four NES records for the year 1665, but three of them are likely to be related to the same event in late February, and one to Aug. 27, thus yielding two different observations. These events again fall into gaps in direct telescopic observations with only generic statements available. For this year, only nine daily direct telescopic records, evenly spread over the second half-year, exist. However, the observers Hevelius and Mezzavacca, both claimed the absence of spots. As a result, the exact level of activity for this year is therefore unknown. Probably, there was some activity in 1665 but not high, owing to direct no-spots records (see. Sect. 2.2.2).

Another NES was reported during three days in mid-March 1684, which falls on no-spot records by la Hire. That year was well covered by telescopic observation, especially in the middle and late year, and it was relatively active $R_{G}=11.7$ (Vaquero et al. 2015a, "strict" model). Taking the probably missed spot in March into account would raise the annual GSN value of this otherwise well-observed year by less than two.

There is additional NES record for the year 1709 (no date or even season given). That year was well observed by different atronomers, with some weak activity reported intermittently throughout the year. The mean annual GSN in the "strict" model is $R_{G}=5.3$.

Thus, except for the year 1684, there is no direct clash between the East-Asian NES records and European telescopic observations, and the former do not undermine the low levels of solar activity suggested by the latter.

\section{Indirect proxy data}

\subsection{Aurorae borealis}

\subsubsection{Geomagnetic observations}

In recent years we have learned a great deal from geomagnetic observations about centennial-scale solar variability and how it influences the inner heliosphere, and hence the Earth (Lockwood et al. 1999; Lockwood 2013). Such studies cannot tell us directly about the MM because geomagnetic activity was first observed in 1722 by George Graham in London and the first properly-calibrated magnetometer was not introduced until 1832 (by Gauss in Göttingen). Graham noted both regular diurnal variations and irregular changes during the peak of solar cycle \#-3 (ca. 1720), which was the first significant cycle after the MM. This raises an interesting question: were these observations made possible by Graham's improvements to the compass needle with its bearing and observation technique or had magnetic activity not been seen before because it had not been strong enough? In any case, despite coming too late to have direct bearing on understanding the MM, the historic geomagnetic data have been extremely important because they have allowed us to understand and confirm the link between sunspot numbers and cosmogenic isotope data. In particular, they have allowed modelling of the open solar flux which shows that the low sunspot numbers in the MM are quantitatively (and not just qualitatively) consistent with the high cosmogenic isotope abundances (Solanki et al. 2000; Owens et al. 2012; Lockwood \& Owens 2014). This understanding has allowed the analysis presented in Sect. 3.4.

\subsubsection{Surveys of historic aurorae}

Earlier in the same solar cycle as Graham's first geomagnetic activity observations, on the night of Tuesday Mar. 17, 1716 
(Gregorian calendar: note the original paper gives the Julian date in use of the time which was Mar. 6), auroral displays were seen across much of northern Europe, famously reported by Edmund Halley (1716) in Great Britain.

What is significant about this event is that very few people in the country had ever seen an aurora before (Fara 1996). Indeed, Halley's paper was commissioned by the Royal Society for this very reason. This event was so rare it provoked a similar review under the auspices of l'Académie des Sciences of Paris (by Giacomo Filippo Maraldi, also known as Jacques Philippe Maraldi) and generated interest at the Royal Prussian Academy of Sciences in Berlin (by Gottfried Wilhelm Leibnitz). All these reviews found evidence of prior aurorae, but none in the previous half century.

Halley himself had observed the 1716 event (and correctly noted that the auroral forms were aligned by the magnetic field) but had never before witnessed the phenomenon. It is worth examining his actual words: “... [of] all the several sorts of meteors [atmospheric phenomena] I have hitherto heard or read of, this [aurora] was the only one I had not as yet seen, and of which I began to despair, since it is certain it hath not happen'd to any remarkable degree in this part of England since I was born [1656]; nor is the like recorded in the English Annals since the Year of our Lord 1574". This is significant because Halley was an observer of astronomical and atmospheric phenomena who even had an observatory constructed in the roof of his house in New College Lane, Oxford where he lived from 1703 onwards. In his paper to the Royal Society, Halley lists reports of the phenomenon, both from the UK and abroad, in the years 1560, 1564, 1575, 1580, 1581 (many of which were reported by Brahe in Denmark), 1607 (reported in detail by Kepler in Prague) and 1621 (reported by Galileo in Venice and Gassendi in Aix, France). Strikingly, thereafter Halley found no credible reports until 1707 (Rømer in Copenhagen and Maria and Gottfried Kirch in Berlin) and 1708 (Neve in Ireland). He states "And since then [1621] for above 80 years, we have no account of any such sight either from home or abroad". This analysis did omit some isolated sightings in 1661 from London (reported in the Leipzig University theses by Starck and Früauff). In addition to being the major finding of the reviews by Halley, Miraldi and others (in England, France and Germany), a similar re-appearance of aurorae was reported in 1716-1720 in Italy and in New England (Siscoe 1980).

The absence of auroral sightings in Great Britain during the $\mathrm{MM}$ is even more extraordinary when one considers the effects of the secular change in the geomagnetic field. For example, using a spline of the IGRF (International Geomagnetic Reference Field ${ }^{4}$ model after 1900 with the gufm 1 model (Jackson et al. 2000) before 1900 we find the geomagnetic latitude of Halley's observatory in Oxford was $60.7^{\circ}$ in 1703 and Edinburgh was at $63.4^{\circ}$. Auroral occurrence statistics were taken in Great Britain between 1952 and 1975, and of these years the lowest annual mean sunspot number was 4.4 in 1954. Even during this low solar activity year there were 169 auroral nights observed at the magnetic latitude that Edinburgh had during the MM and 139 at the magnetic latitude that Oxford had during the MM (Paton 1959). In other words, the British Isles were at the ideal latitudes for observing aurora during the $\mathrm{MM}$ and yet the number reported was zero. This is despite some careful and methodical observations revealed by the notebooks of several scientists: for example, Halley's notebooks regularly and repeatedly use the

\footnotetext{
4 http://www.ngdc.noaa.gov/IAGA/vmod/igrf.html
}

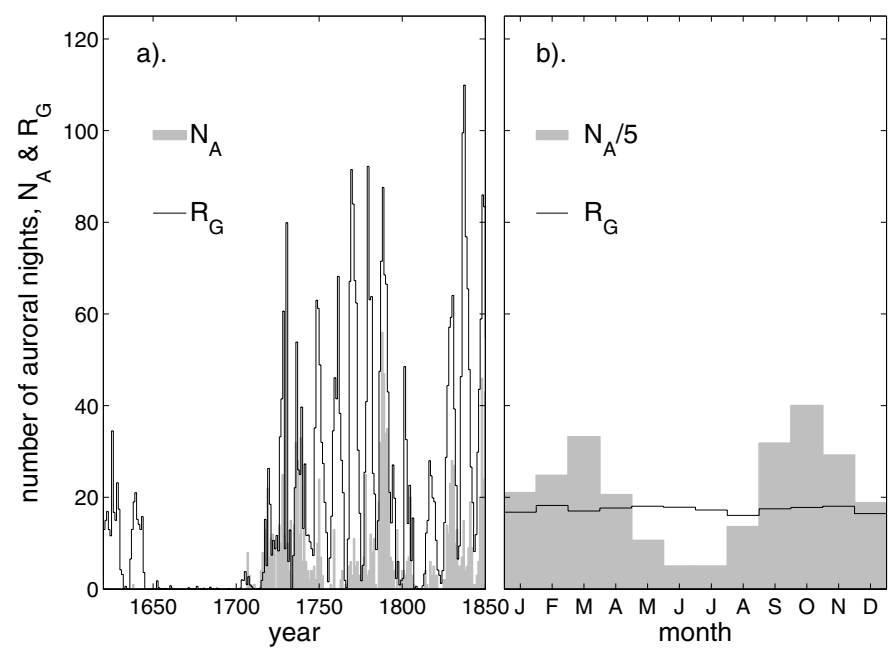

Fig. 10. Panel A) number of auroral nights, $N_{\mathrm{A}}$, in calendar years for observations in Great Britain collated by E. J. Lowe (1870) with the addition of the observations by Thomas Hughes (Harrison 2005) and John Dalton (Dalton 1834) (grey histogram). Annual group sunspot number of Hoyt \& Schatten (1998), with the adoption of recent corrections by Vaquero et al. (2011) and Vaquero \& Trigo (2014) (black line). Lowes personal copy of his catalogue of natural phenomena (including auroras) was only recently discovered and was compiled completely independently of other catalogues. Yet it shows, like the others, the dearth of sightings during the MM, some events in 1707 and 1708 and the return of regular sightings in 1716. Panel B) annual variation of $N_{\mathrm{A}}$ in the same dataset and of $R_{G}$.

term "clear skies" which make it inconceivable that he would not have noted an aurora had it been present.

Halley's failure to find auroral sightings in the decades before 1716 is far from unique. Figure 10 is a plot of auroral occurrence in Great Britain from a previously unknown source. It is shown with the group sunspot number $R_{G}$ during the MM. This catalogue of auroral sightings in the UK was published in 1870 by an astronomer and a fellow of the Royal Society, E. J. Lowe, who used parish records, newspaper reports and observations by several regular observers. His personal copy of the book (with some valuable "marginalia" - additional notes written in the margin) was recently discovered in the archives of the Museum of English Rural Life at the University of Reading, UK (Lowe 1870). Here we refer to this personally commented copy of the book. We have added the observations listed in the diary of Thomas Hughes in Stroud, England (Harrison 2005) and the observations made by John Dalton in Kendall and later Manchester (Dalton 1834) to Lowe's catalogue of English recordings. Like so many other such records, the time series of the number of auroral nights during each year, shown by the grey histogram in Fig. 10a, reveals a complete dearth of auroral sightings during the MM. As such, this record tells us little that is not known from other surveys; however, it is important to note that this compilation was made almost completely independently of, and using sources different from other catalogues such as those by de Mairan and Fritz (see below).

Figure $10 \mathrm{~b}$ shows the annual variation of the number of auroral nights and reveals the semi-annual variation (Sabine 1852) (equinoctial peaks in auroral occurrence were noted by de Mairan 1733). A corresponding semi-annual variation in geomagnetic activity (Sabine 1852; Cortie 1912) is mainly caused by the effect of solar illumination of the nightside auroral current electrojets (Cliver et al. 2000; Lyatsky et al. 2001; Newell et al. 2002), leading to equinoctial maxima in geomagnetic activity. 
However, lower-latitude aurorae are caused by the inner edge of the cross-tail current sheet being closer to the Earth, caused by larger open flux in the magnetosphere-ionosphere system (Lockwood 2013) and so are more likely to be caused by the effect of Earth's dipole tilt on solar wind-magnetosphere coupling and, in particular the magnetic reconnection in the magnetopause that generates the open flux (Russell \& McPherron 1973). This is convolved with a summer-winter asymmetry caused by the length of the annual variation in the dark interval in which sightings are possible. Note that Fig. 10b shows a complete lack of any annual variation in group sunspot number, as expected. This provides a good test of the objective nature of the combined dataset used in Fig. 10. Both parts a and b of Fig. 10 are very similar in form to the corresponding plots made using all the other catalogues.

Elsewhere, however, other observers in 1716 were familiar with the phenomenon of aurorae (Brekke \& Egeland 1994). For example Joachim Ramus, a Norwegian (born in Trondheim in 1685 but by then living in Copenhagen), also witnessed aurora in March 1716, but unlike Halley was already familiar with the phenomenon. Suno Arnelius in Uppsala had written a scientific thesis on the phenomenon in 1708. Indeed after the 1707 event Rømer had noted that, although very rare in his native Copenhagen, such events were usually seen every year in Iceland and northern Norway (although it is not known on what basis he stated this) (Stauning 2011; Brekke \& Egeland 1994). But even at Nordic latitudes aurorae appear to have been relatively rare in the second half of the 17th century (Brekke \& Egeland 1994). Petter Dass, a cleric in Alstahaug, in middle Norway, who accurately and diligently reported everything he observed in the night sky between 1645 and the time of his death in 1707, and who had read many historic reports of aurorae, never once records seeing them himself. In his thesis on aurorae (completed in 1738), Peter Møller of Trondheim argues that the aurora reported over Bergen on New Year's Eve 1702 was the first that was ever recorded in the city. Celsius in Uppsala was 15 years old at the time of the March 1716 event but subsequently interviewed many older residents of central Sweden and none had ever seen an aurora before. Johann Anderson was the mayor of Hamburg and discussed aurorae with Icelandic sea captains. They told him that aurorae were seen before 1716 , but much less frequently (reported in Horrebow 1752). An important contribution to the collation of reliable auroral observations was written in 1731 by Jean-Jacques d'Ortous de Mairan (de Mairan 1733), with a second edition published in 1754 . Both editions are very clear in that aurorae were rare for at least 70 years before their return in 1716. The more thorough surveys by Lovering (1860), Fritz $(1873,1881)$ and Link $(1964,1978)$ have all confirmed this conclusion (see Eddy 1976).

\subsubsection{Reports of aurorae during the Maunder minimum}

The above does not mean that auroral sighting completely ceased during the MM. de Mairan's original survey reported 60 occurrences of aurorae in the interval 1645-1698. Many authors have noted that the solar cycle in auroral occurrence continued during the MM (Link 1978; Vitinskii 1978; Gleissberg 1977; Schröder 1992; Legrand et al. 1992). One important factor that must be considered in this context is the magnetic latitude of the observations. It is entirely possible that aurora were always present, at some latitude and brightness, and that the main variable with increasing solar activity is the frequency of the equatorward excursions of brighter forms of aurorae. In very quiet times, the aurora would then form a thin, possibly fainter, band at very high latitudes, greatly reducing the chance of observation. An important indication that this was indeed the case comes from a rare voyage into the Arctic during the MM by the ships Speedwell and Prosperous in the summer of 1676. This was an expedition approved by the then secretary to the British Admiralty, Samuel Pepys, to explore the north east passage to Japan. Captained by John Wood, the ships visited northern Norway and Novaya Zemlya (an Arctic archipelago north of Russia), reaching a latitude of $75^{\circ} 59^{\prime}$ (geographic) before the Speedwell ran aground. Captain Wood reported that aurorae were only seen at the highest latitudes by a local seaman that he met. Furthermore, anecdotal evidence was supplied by Fritz who quoted a book on Greenland fisheries that aurorae were sometimes seen in the high Arctic at this time. The possibility of aurora watching at very high latitudes was the main criticism of de Mairan's catalogue by Ramus, claiming that it relied on negative results from expeditions that were outside the observing season set by sunlight (Brekke \& Egeland 1994; Stauning 2011).

\subsubsection{Comparison of aurorae during the Maunder and Dalton minima}

The debate about the reality of the drop in auroral occurrence during the MM was ended when a decline was seen during the DM (c. 1790-1830). This minimum is seen in all the modern catalogues mentioned above and in others, such as that by Nevanlinna (1995) from Finnish observatories, which can be calibrated against modern-day observations (Nevanlinna \& Pulkkinen 2001). Many surveys show the MM to be deeper than the DM in auroral occurrence but not by a very great factor (e.g., Silverman 1992). However, given the likelihood that aurorae were largely restricted to a narrow band at very high latitudes during both minima, observations at such high latitudes become vital in establishing the relative depths of these two minima. In this respect the survey by Vázquez et al. (2014) is particularly valuable as, in addition to assigning locations to every sighting, it includes the high latitude catalogues by Rubenson (1882) and Tromholt (1898) as well as those of Silverman (1992) and Fritz (1873). The quality control employed by Vázquez et al. (2014) means that their survey extends back to only 1700 which implies that it covers 15 years before the events of 1716 and hence only the last solar cycle of the MM.

Figure 11 is an analysis of the occurrence of aurorae between 1700 and 1900. The green line is the number of auroral nights per year at geomagnetic latitudes below $56^{\circ}$ from a combination of the catalogues of Nevanlinna (1995), Fritz (1873), Fritz (1881) and Legrand \& Simon (1987). This sequence clearly shows that aurorae at geomagnetic latitudes below $56^{\circ}$ were indeed rarer in both the last cycle of the MM and the two cycles of the DM. However, the number of recorded auroral sightings was significantly greater during DM than that in the MM. The points in Fig. 11 show the geomagnetic latitude and time of auroral sightings from the catalogue of Vázquez et al. (2014) (their Fig. 9). Black diamonds, red squares and red triangles are, respectively, to indicate observing sites in Europe and North Africa, North America, and Asia. Blue dashed lines mark the minimum latitude of auroral reports in the last solar cycle of the MM and in the two cycles of the DM. During the DM many more aurorae were reported (symbols in the figure) poleward of the $56^{\circ}$ latitude.

Considerably fewer arcs were reported at the end of the MM at these latitudes, despite the inclusion by Vázquez et al. (2014) of two extra catalogues of such events for this period at auroral oval latitudes. Furthermore, the two dashed lines show the 


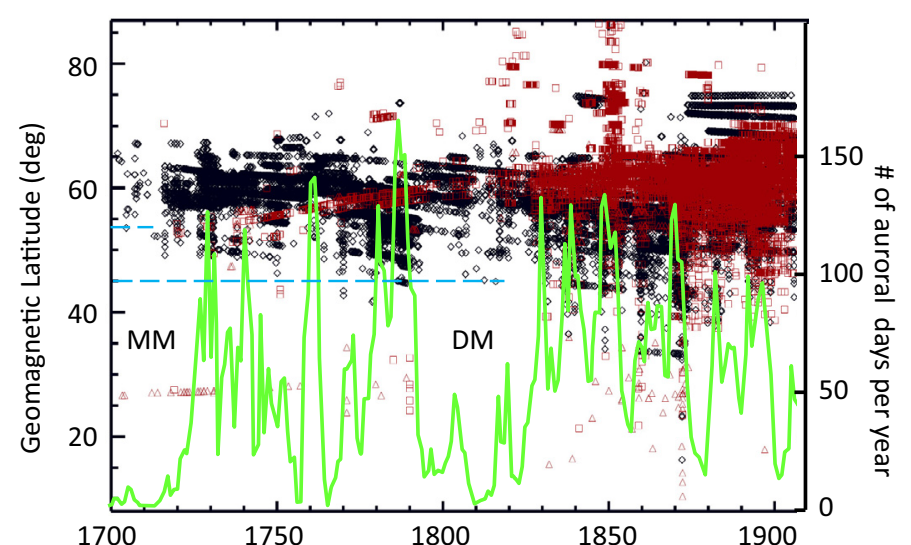

Fig. 11. Occurrence of auroral reports, 1700-1900. The green line is the number of auroral nights at geomagnetic latitudes below $56^{\circ}$ from a combination of several catalogues (Nevanlinna 1995; Fritz 1873, 1881; Legrand \& Simon 1987). The points show the geomagnetic latitude and time of auroral sightings from the catalogue of Vázquez et al. (2014) (their Fig. 9). Black diamonds, red squares and red triangles are, respectively, for observing sites in Europe and North Africa, North America, and Asia. Blue dashed lines mark the minimum latitude of auroral reports in the last solar cycle of the MM and in the two cycles of the DM.

minimum latitude of events seen in these two minima: whereas events were recorded down to a magnetic latitude of $45^{\circ}$ in the $\mathrm{DM}$, none were seen at the end of the MM below $55^{\circ}$, consistent with the dearth of observations in central Europe at the time. Note that during the MM there are some observations at magnetic latitudes near $27^{\circ}$, all from Korea (with one exception which is from America). They were reported as being observed in all directions, including the South, and to be red (Yau et al. 1995) which makes them unfavorable candidates for classical aurorae. By their features these could have been stable auroral red (SAR) arcs (Zhang 1985) which in modern times are seen at mid-latitudes mainly during the recovery phase of geomagnetic storms (Kozyra et al. 1997). These arcs are mainly driven by the ring current and differ from normal auroral phenomena. Moreover, as stated by Zhang (1985) "We cannot rule out the possibility that some of these Korean sightings were airglows and the zodiacal light". Here we concentrate on the higher latitude auroral oval arcs. The plot also shows the return of reliable lower latitude sightings in Europe in 1716 and in America in 1718.

Figure 12 corresponds to Fig. 10, but now based on a compilation of all major historical auroral catalogues. Figure $12 \mathrm{em}-$ ploys the list of aurora days by Křivský \& Pejml (1988) which is based on 39 different catalogues of observations at geomagnetic latitudes below $55^{\circ}$ in Europe, Asia, and North Africa. To this has been added the catalogue of Lovering $(1867)^{5}$ with observations made in and around Cambridge, Massachusetts, USA which was at a magnetic latitude close to $55^{\circ}$ in 1900 , and the recently-discovered catalogue of observations from Great Britain by Lowe (1870). Figure 12a shows the low level and gradual decline in the occurrence of low- and mid-latitude auroral observations during the MM and in the decades leading up to it. This is in contrast to the general rise in observation reports that exists on longer timescales as scientific recording of natural phenomena became more common. After the MM, solar cycles in the auroral occurrence can clearly be seen and the correlation with the annual mean group sunspot numbers $R_{G}$ is clear. Even

\footnotetext{
5 Paper and catalogue are available from http://www.jstor.org/ stable/25057995
}

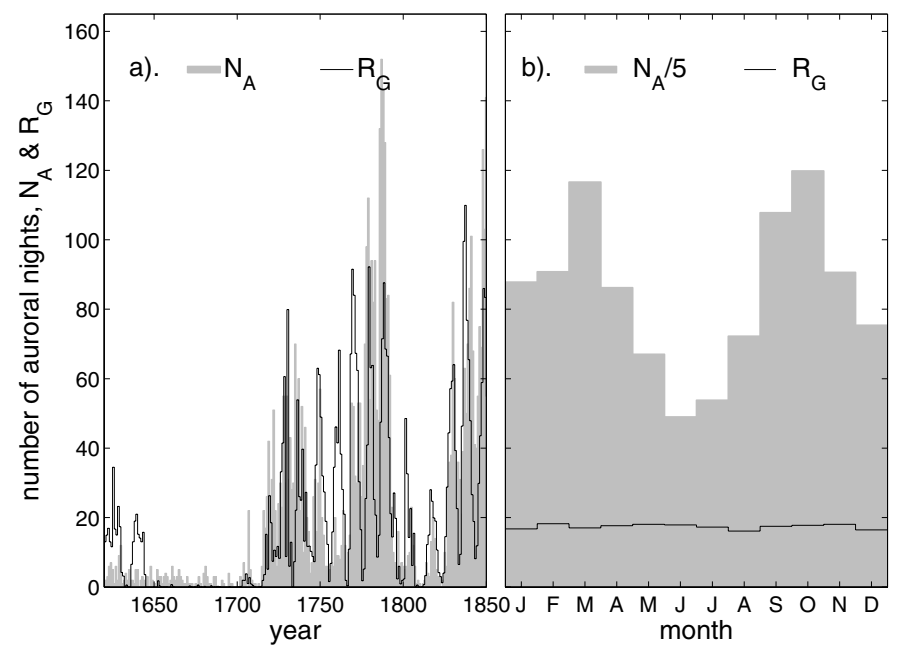

Fig. 12. Same as Fig. 10, but compiled from 41 different catalogues of auroral observations at magnetic latitudes below 55 in Europe, Asia, North Africa, New England and Great Britain. The time series covers both the Maunder and the Dalton minima.

for these lower latitude auroral observations it is unquestionable that the MM is considerably deeper than the later DM. The annual variability (Fig. 12b) is obvious also for this dataset.

From all of the above it is clear that the MM in auroral, and hence solar, activity was a considerably deeper minimum than the later DM.

\subsection{Solar corona during the Maunder minimum}

As shown already by Eddy (1976) and re-analyzed recently by Riley et al. (2015), recorded observations of solar eclipses suggest the virtual absence of the bright structured solar corona during the MM. While 63 total solar eclipses should had taken place on Earth between 1645 and 1715, only four (in years 1652, 1698, 1706 and 1708) were properly recorded in a scientific manner others were either not visible in Europe or not described in sufficient detail. These reports (see details in Riley et al. 2015) suggest that the solar corona was reddish and unstructured, which was interpreted (Eddy 1976) as the F-corona (or zodiacal light) in the absence of the $\mathrm{K}$ corona. The normally structured corona reappeared again between 1708 and 1716, according to later solar eclipse observations, as discussed in Riley et al. (2015).

Observations of the solar corona during total eclipses, although rare and not easy to interpret, suggest that the corona was very quiet and had shrunk during the MM, with no large scale structures such as streamers. This also implies the decline of surface activity during the MM.

\subsection{Heliospheric conditions}

The Sun was not completely quiet during the MM, and a certain level of heliospheric activity was still present - the heliosphere existed, the solar wind was blowing, the heliospheric magnetic field was there, although at a strongly reduced level (e.g. Cliver et al. 1998; Caballero-Lopez et al. 2004; McCracken \& Beer 2014). Since heliospheric disturbances, particularly those leading to cosmic ray modulation, are ultimately driven by solar surface magnetism (Potgieter 2013), and this is also manifested through sunspot activity, cosmic ray variability is a good indicator of solar activity, especially on time scales longer than a solar cycle (Beer 2000; Beer et al. 2012; Usoskin 2013). Here 


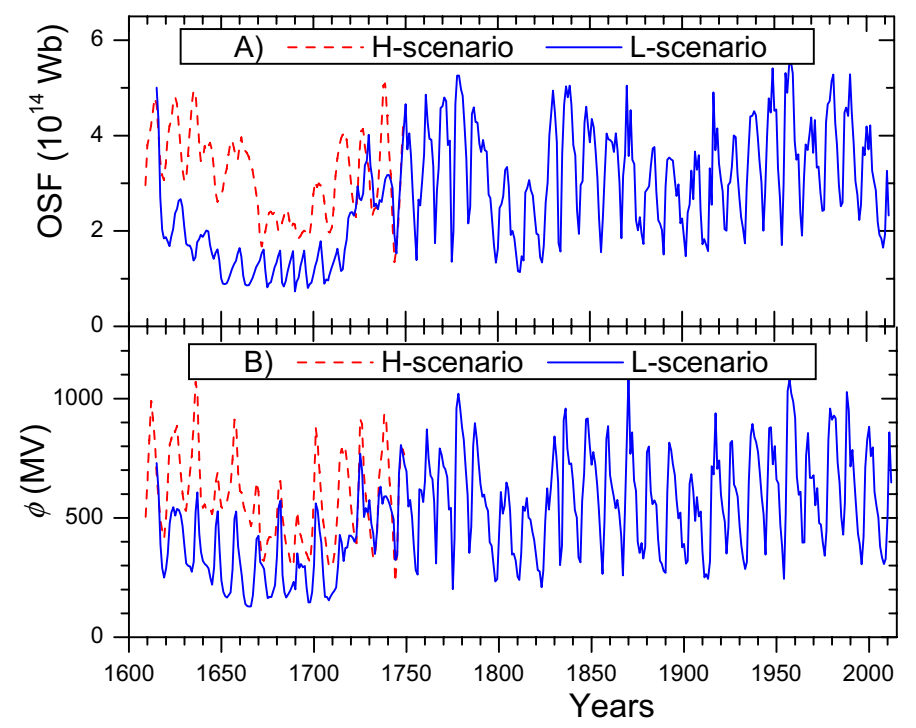

Fig. 13. Evolution with time of the open solar magnetic flux, OSF, (panel A)) and of the modulation potential $\phi$ (panel B)) for the two scenarios of solar activity during the MM (see text).

we estimate the heliospheric conditions evaluated for the period around the MM, using different scenarios of solar activity, and compare these with directly measured data on cosmogenic isotopes in terrestrial and extra-terrestrial archives.

The open solar magnetic flux (OSF) is one of the main heliospheric parameters defining the heliospheric modulation of cosmic rays. It is produced from surface magnetic fields expanding into the corona from where they are dragged out into the heliosphere by solar wind. Consequently, it can be modelled using the surface distribution of sunspots and a model of the surface magnetic flux transport (Wang \& Sheeley 2002). If their exact surface distribution is not known, the number of sunspots can also serve as a good input to OSF computations, using models of magnetic flux evolution (Solanki et al. 2000, 2002; Lockwood \& Owens 2014) or with more complex surface flux transport simulations (e.g., Jiang et al. 2011b). Here we use the simpler, but nonetheless very successful, model to calculate the OSF from the sunspot number series (Lockwood \& Owens 2014; Lockwood et al. 2014a). This model quantifies the emergence of open flux from sunspot numbers using an analysis of the occurrence rate and magnetic flux content of coronal mass ejections as a function of sunspot numbers over recent solar cycles (Owens \& Lockwood 2012). The open flux fractional loss rate is varied over the solar cycle with the current sheet tilt, as predicted theoretically by Owens et al. (2011) and the start times of each solar cycle taken from sunspot numbers (during the MM the ${ }^{10} \mathrm{Be}$ cycles are used). The one free parameter needed to solve the continuity equation, and so model the OSF, is then obtained by fitting to the open flux reconstruction derived from geomagnetic data for 1845-2012 by Lockwood et al. (2014a).

We computed OSF series (Fig. 13) corresponding to the two scenarios for the number of sunspots during the MM (see Sect. 2), viz. L- and H-scenarios. The OSF is the main driver of the heliospheric modulation of cosmic rays on timescales of decades to centuries (e.g., Usoskin et al. 2002), with additional variability defined by the heliospheric current sheet (HCS) tilt and the large scale polarity of the heliospheric magnetic field (Alanko-Huotari et al. 2006; Thomas et al. 2014). Using an updated semi-empirical model (Alanko-Huotari et al. 2006; Asvestari \& Usoskin 2015), we have computed the modulation

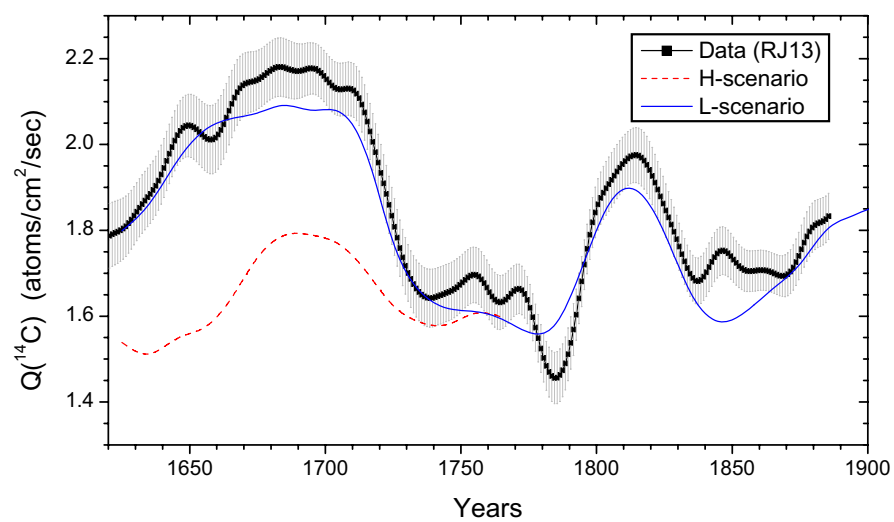

Fig. 14. Time profile of decadally smoothed radiocarbon ${ }^{14} \mathrm{C}$ production rate. The black curve with grey error bars represents the reconstruction by Roth \& Joos (2013) based on the Intcal13 (Reimer et al. 2013) radiocarbon data. Coloured curves depict the computed production for the two scenarios as labeled in the legend (see text for details).

potential $\phi$ (see definition and formalism in Usoskin et al. 2005) for the period since 1610 for the two scenarios described above, as shown in Fig. 13b. These series will be used for subsequent analysis.

\subsection{Cosmogenic radionuclides}

The cosmogenic radionuclides are produced by cosmic rays in the atmosphere, and this forms the dominant source of these isotopes in the terrestrial system (Beer et al. 2012). Production of the radionuclides is controlled by solar magnetic activity quantified in the heliospheric modulation potential (see above) and by the geomagnetic field, both affecting the flux of galactic cosmic rays impinging on Earth. For independently known parameters of the geomagnetic field, the measured abundance of cosmogenic radioisotopes in a datable archive can be used to reconstruct solar and/or heliospheric magnetic activity in the past. This is done applying proper modelling, including the production and transport of the isotopes in the Earths atmosphere (Beer et al. 2012; Usoskin 2013). Here we use a recent archeomagnetic reconstruction of the geomagnetic field (Licht et al. 2013) before 1900. In the subsequent subsections we apply the solar modulation potential series obtained for the two scenarios (Fig. 13b) to cosmogenic radionuclides.

\subsection{1. ${ }^{14} \mathrm{C}$ in tree trunks}

Using the recent model of radiocarbon ${ }^{14} \mathrm{C}$ production (Kovaltsov et al. 2012), we have computed the expected global mean radiocarbon production rate for the two scenarios analyzed here, as shown in Fig. 14. One can see that the variability of ${ }^{14} \mathrm{C}$ production is quite different in the $\mathrm{H}$-(red dotted) and $\mathrm{L}$-(blue solid curve) scenarios. While the former is rather constant, with only a weak maximum during the MM, and even smaller than that for the DM in the early 1800s, the latter exhibits a high and long increase during the MM, which is significantly greater than that for the DM in both amplitude and duration.

In the same plot we show also the ${ }^{14} \mathrm{C}$ production rate obtained by Roth \& Joos (2013) from the Intcal13 (Reimer et al. 2013) global radiocarbon data, using a new generation stateof-the-art carbon cycle model. The ${ }^{14} \mathrm{C}$ global production expected for the L-scenario matches the data very well given the uncertainties, over the entire period of 1610-1880 and confirms 


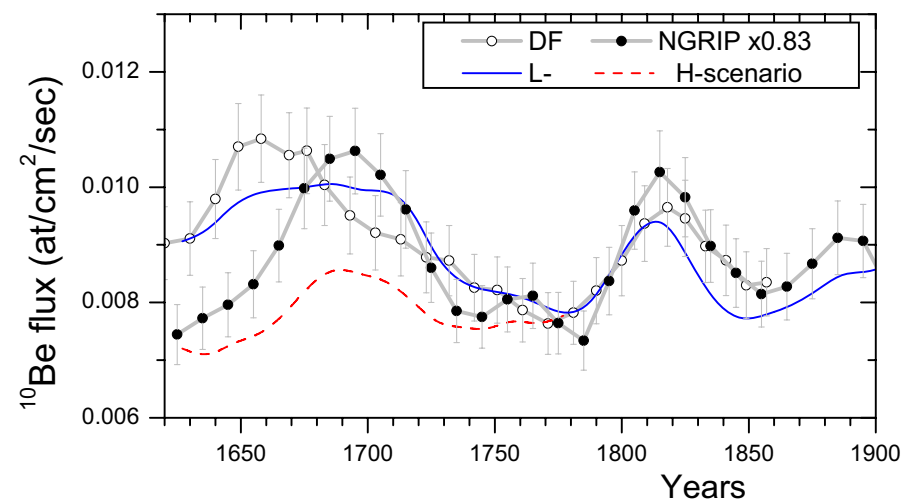

Fig. 15. Depositional flux of ${ }^{10} \mathrm{Be}$ in polar ice. All data are pseudodecadal and 25-yr low-pass smoothed because of strong high-frequency noise. Grey curves depict the fluxes measured at Dome Fuji (DF) in Antarctica (Horiuchi et al. 2007), and at NGRIP (scaled as 0.83), Greenland (Berggren et al. 2009). Error bars are estimates of both statistical and systematic errors. The blue and red curves depict the modelled ${ }^{10} \mathrm{Be}$ depositional fluxes for the $\mathrm{L}$ - and $\mathrm{H}$-scenarios (see text for details), respectively.

the validity of this scenario. On the contrary, the H-scenario, both quantitatively and qualitatively, disagrees with the observed production during the MM, implying that the solar modulation of cosmic rays is grossly overestimated during the MM by this scenario. Thus, the ${ }^{14} \mathrm{C}$ data support a very low level of heliospheric (and hence solar surface magnetic) activity during the $\mathrm{MM}$, a level that is considerably lower than during the DM.

\subsection{2. ${ }^{10} \mathrm{Be}$ in polar ice cores}

With a similar approach to the one taken for the analysis of ${ }^{14} \mathrm{C}$ (Sect. 3.4.1) we have computed the depositional flux of ${ }^{10} \mathrm{Be}$ in polar regions. We used the same archeomagnetic model (Licht et al. 2013), the recent ${ }^{10} \mathrm{Be}$ production model by Kovaltsov \& Usoskin (2010), and the atmospheric transport/deposition model as parameterized by Heikkilä et al. (2009).

The results are shown in Fig. 15. As discussed in the previous section, the expected curve for the H-scenario (red dashed curve) shows little variability, being lower (implying higher solar modulation) during the MM than during the DM, while the L-scenario yields a higher flux (lower modulation) during the MM. The two grey curves depict ${ }^{10} \mathrm{Be}$ fluxes measured in two opposite polar regions. One is the data series of ${ }^{10} \mathrm{Be}$ depositional flux measured in the Antarctic Dome Fuji (DF) ice core (Horiuchi et al. 2007). The other is the ${ }^{10} \mathrm{Be}$ flux series measured in the Greenland NGRIP (North Greenland Ice-core Project) ice core (Berggren et al. 2009). Because of the different local climate conditions (Heikkilä et al. 2009), the latter was scaled by a factor of 0.83 to match the same level. This scaling does not affect the shape of the curve and in particular not the ratio of the ${ }^{10} \mathrm{Be}$ flux in the MM and the DM. One can see that while the time profiles of the two datasets differ in detail, probably because of the different climate patterns (Usoskin et al. 2009a) and/or timing uncertainties, both yield high ${ }^{10} \mathrm{Be}$ production during the MM. This corresponds to extremely low solar activity (McCracken et al. 2004). The L-scenario agrees with the data reasonably well (the data display even higher maxima than the model), while the H-scenario clearly fails to reproduce the variability of ${ }^{10} \mathrm{Be}$ measured in polar ice.

Thus, the ${ }^{10} \mathrm{Be}$ data from both Antarctic and Greenland ice cores support a very low level of heliospheric (and hence solar

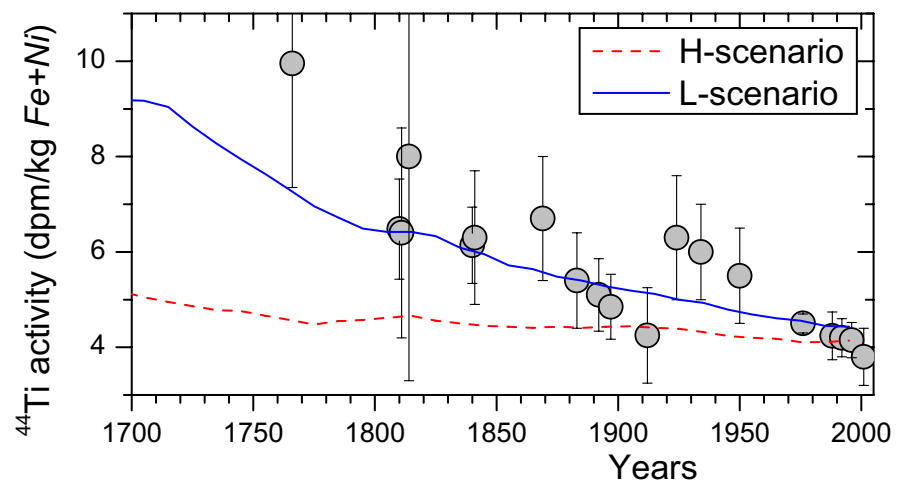

Fig. 16. Time profile of the ${ }^{44} \mathrm{Ti}$ activity in units of disintegrations per minute per $\mathrm{kg}$ of iron and nickel in the meteorite. The grey dots with error bars are the measurements (Taricco et al. 2006). The coloured curves depict the computed activity for the two scenarios (see text).

surface magnetic) activity during the MM, significantly lower than during the DM.

\subsection{3. ${ }^{44} \mathrm{Ti}$ in meteorites}

While records of terrestrial cosmogenic radionuclides may be affected by transport and deposition processes, which are not always exactly known (Usoskin et al. 2009a; Beer et al. 2012), cosmogenic nuclides measured in fallen meteorites are free of this uncertainty, since the nuclides are produced directly in the meteorite's body in space, and measured after their fall to Earth. However, time resolution is lost, or at least greatly reduced in this case, and the measured activity represents a balance between production and decay over the time before the fall of the meteorite. An ideal cosmogenic nuclide for our purpose is ${ }^{44} \mathrm{Ti}$ with a half-life of about 60 years (Ahmad et al. 1998; Bonino et al. 1995). Here we test the cosmic ray variability as inferred from different scenarios of solar activity since 1600 , following exactly the method described in detail in Usoskin et al. (2006) and the dataset of ${ }^{44} \mathrm{Ti}$ activity measured in 19 stony meteorites that fell between 1776 and 2001 (Taricco et al. 2006). Applying the modulation potential series as described in Sect. 3.3 to the cosmic ray flux and calculating the expected ${ }^{44} \mathrm{Ti}$ activity as a function of the time of the meteorite's fall, we compare the model computations for the two scenarios with the measured values in Fig. 16.

One can see that the series for the L-scenario fits the data rather well, whereas the curve resulting from the H-scenario lies considerably too low. As a merit parameter of the agreement we use the $\chi^{2}$ value for the period of 3.3 half-lives (attenuation factor 10) since the middle of the MM, viz. until 1880, which includes seven meteorites that fell between 1776 and 1869 ( 7 degrees of freedom). The $\chi^{2}(7)$ for the L-scenario is 2 ( 0.33 per degree of freedom), which perfectly fits the hypothesis. For the H-scenario, however, $\chi^{2}(7)=17.6$ (2.93 per degree of freedom), which indicates that this hypothesis should be rejected with a high significance of 0.014 . Note that the long residence times of the meteorites within the helisophere means that the difference during the MM between the two scenarios has an influence on the predicted ${ }^{44} \mathrm{Ti}$ abundances even for meteorites that fell to Earth relatively recently. This results in the observed abundances being inconsistent with the $\mathrm{H}$-scenario for a large number of the meteorites whereas they are consistent, within the observational uncertainty, with the L-scenario.

Accordingly, the hypothesis of a high level of solar activity during the MM is rejected at a high significance level using 
indirect data of ${ }^{44} \mathrm{Ti}$ in meteorites, while the conventional scenario of very low activity during the MM is in full agreement with the data.

\section{Consequences of the Maunder minimum}

\subsection{Solar dynamo}

Major changes in the secular level of solar activity, such as grand minima/maxima form a challenge for our understanding of the origin and evolution of the solar magnetic field. It has recently been shown, by analyzing the sunspot numbers reconstructed from ${ }^{14} \mathrm{C}$ for the last 3000 years, that grand minima form a separate mode of solar activity (Usoskin et al. 2014), which likely corresponds to a special regime of the solar dynamo. In addition to the traditional concept of cyclic solar activity associated with periodic nonlinear oscillations of large-scale magnetic fields, solar dynamo models now include, as their natural element, various deviations from pure periodicity. Accordingly, it is crucially important for our understanding of solar and stellar dynamos to know whether grand minima indeed exist and what their parameters are. Although a possibility of direct modelling of the MM by dynamo theory is limited by the lack of information concerning flows in the solar interior, some important observational results pointing to quite peculiar features of the solar surface magnetic field configuration (slower, but more differential, rotation, strong hemispheric asymmetry of sunspot formation, and a possibly variable solar diameter) during the MM have been found (Ribes \& Nesme-Ribes 1993; Sokoloff \& Nesme-Ribes 1994).

Most large-scale solar dynamo models operate with averaged quantities taken as statistical ensembles of a moderate number of convective cells (see, e.g., a review by Charbonneau 2010) and include, in addition to the solar differential rotation, the collective inductive effect of mirror asymmetric convective turbulence and/or meridional circulation. The observational knowledge of the turbulent quantities is especially limited, and they have to be estimated using local direct numerical simulations (e.g. Schrinner et al. 2005; Käpylä et al. 2009). Generally, fluctuations of the intensity of the main drivers of the dynamo of up to $10-20 \%$ are expected. By including such fluctuations into a mean-field dynamo model it is possible (e.g. Moss et al. 2008b; Choudhuri \& Karak 2012; Passos et al. 2014) to reproduce numerically a dynamo behaviour which deviates from the stable cyclic evolution and depicts variability like the grand minima. It is important that asymmetric magnetic configuration modes of mixed parity with respect to the solar equator can be excited even in the framework of a conventional $\alpha \Omega$ dynamo (Brandenburg et al. 1989; Jennings \& Weiss 1991), and similar to that existing on the Sun at the end of the MM (Sokoloff \& Nesme-Ribes 1994). The asymmetric sunspot occurrence during the late MM may be a signature of an unusual mode of the dynamo. It is known for spherical dynamos (Moss et al. 2008a) that even relatively moderate deviations from the nominal parameters associated with normal cycles can lead to the excitation of specific magnetic configurations, for example, with a quadrupolar symmetry with respect to the solar equator. However, this kind of asymmetry is not expected for regular cycles with normal values of the driving parameters. This, along with the suppression of the cycle amplitude, may be a specific feature of a MM-type event.

An interesting fact is that the solar surface rotation was reported to be slower and more differential (changing faster with latitude) during the second half of the MM than during modern times (Ribes \& Nesme-Ribes 1993). The enhanced differential rotation may have lasted until the mid-17th century (Arlt \& Fröhlich 2012). These facts are related to the operation of the solar dynamo during the MM. Since the solar differential rotation is a main driver of the dynamo, and the asymmetry implies a specific configuration, the dynamo could have been operating in a special state during this period.

Differential rotation modulation and mixed parity are also an outcome of nonlinear dynamo models including Lorentz forces and the momentum equation. In those cases, grand minima are produced without a stochastic dynamo effect (Küker et al. 1999; Pipin 1999; Bushby 2006, for spherical shells). The presence of grand minima is therefore a natural feature in mean-field dynamo modelling, including "side-effects", such as differential rotation variation and mixed parity, and is a result of stochasticity and the nonlinearity of the full MHD (magnetohydrodynamic) equations.

Thus, the known phenomenology of the MM does not favor its interpretation as just a modulation of the normal 22-year Hale cycle by an additional longer cycle (Gleissberg cycle), such as the one proposed by ZP15. The very asymmetric and suppressed sunspot activity, accompanied by slower differential rotation, during the late phase of the MM implies, in the light of dynamo theory, a special mode of dynamo operation, leading to grand minima.

Meanwhile, it is still difficult to perform a direct numerical modelling of the MM, including sufficiently small scales, to adequately model the convective turbulence (e.g. Charbonneau 2010). Although modern solar-type dynamo models reproduce the "regular" part of the behaviour of the large-scale solar magnetic field (Ghizaru et al. 2010; Brown et al. 2011; Schrinner et al. 2012; Gastine et al. 2012; Käpylä et al. 2012), it is still challenging to extend the integration time over several magnetic cycles as needed to reproduce a Maunder-like minimum (see, however, Passos \& Charbonneau 2014; Augustson et al. 2015). Thus, while the MM is identified as a special mode of the solar dynamo, we are not yet able to precisely model it.

\subsection{Solar irradiance}

It is now widely accepted that variations in solar irradiance in different wavelengths on time scales longer than about a day are driven by changes in the solar surface coverage by magnetic features, such as sunspots that lead to a darkening of the solar disc, and faculae or network elements that lead to a brightening (see, e.g., Domingo et al. 2009; Solanki et al. 2013, and references therein). Consequently, the number of sunspots and faculae present during the MM would affect both the total (TSI) and the spectral (SSI) irradiance of the Sun at that special time in the history of solar activity.

Direct observations of TSI and SSI are available only from 1978 onwards (e.g., Fröhlich 2013; Kopp 2014). Consequently, a number of models have been developed that reconstruct solar irradiance back to the MM. The TSI produced by a selection of such models is plotted in Fig. 17. The selected models are based on very different data and techniques.

Steinhilber et al. (2009) and Delaygue \& Bard (2011) obtained their reconstructions from timeseries of ${ }^{10} \mathrm{Be}$ concentrations in ice (green colour tones in the figure). Both studies used a simple linear regression to calculate TSI. Steinhilber et al. (2009) first estimated the interplanetary magnetic field from the ${ }^{10} \mathrm{Be}$ data and then used a linear regression with the measured TSI following Fröhlich (2009). Delaygue \& Bard (2011) scaled the ${ }^{10} \mathrm{Be}$ record by assuming a fixed change in TSI between the MM and the last decades. Not shown in Fig. 17, and also 


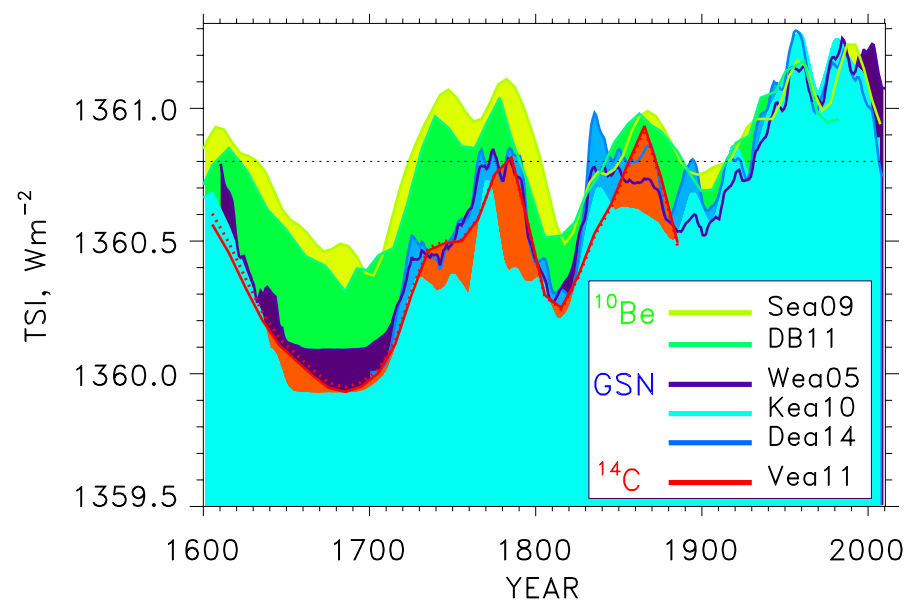

Fig. 17. Selected TSI reconstructions since 1600, labeled in the plot are: Sea09 -Steinhilber et al. (2009); DB11 - Delaygue \& Bard (2011); Wea05 - Wang et al. (2005); Kea10 - Krivova et al. (2010); Dea14 Dasi-Espuig et al. (2014, priv. comm.); Vea11 - Vieira et al. (2011). The green, blue and red colour tones are used for the reconstructions based on the ${ }^{10} \mathrm{Be}$, sunspot and ${ }^{14} \mathrm{C}$ data, respectively. The black dotted line marks the TSI value at modern solar activity minimum conditions according to SORCE/TIM measurements.

relying on the ${ }^{10} \mathrm{Be}$ data, is the TSI reconstruction by Shapiro et al. (2011). The magnitude of the secular change in this model comes from the difference between semi-empirical model atmospheres, describing the darkest parts of the solar surface, and the average quiet Sun (during modern times). The secular change thus obtained is significantly higher than in all other models ( $3 \mathrm{~W} \mathrm{~m}^{-2}$ even after the re-assessment by Judge et al. 2012). However, the shape of the secular change comes again from a linear regression to the ${ }^{10} \mathrm{Be}$ record, so that it is essentially the same as in the other two models using ${ }^{10} \mathrm{Be}$ concentrations.

Blue colour tones show reconstructions that employ more physics-based approaches and are built on the sunspot number. Wang et al. (2005) reconstructed the TSI from the magnetic flux evolved by a flux transport simulation (Sheeley 2005). Krivova et al. (2010) employed the approach of Solanki et al. (2002) to compute the magnetic flux from the sunspot number and therefrom the technique of Krivova et al. (2007) to compute the TSI and the spectral irradiance. Dasi-Espuig et al. (2014) used a flux transport model (Jiang et al. 2010, 2011b) to simulate solar magnetograms to which they applied the SATIRE-S model (Fligge et al. 2000; Krivova et al. 2003).

Finally, Vieira et al. (2011) adapted the models by Solanki et al. (2002) and Krivova et al. (2007) for use with ${ }^{14} \mathrm{C}$ data (red colour tones). The red lines show reconstructions by Vieira et al. (2011) based on two different models of the geomagnetic field. They hardly diverge over the period in consideration.

The magnitude of the secular increase in the TSI since the MM differs by roughly $0.5 \mathrm{~W} \mathrm{~m}^{-2}$ between the models shown in the figure. The full range of this variation is actually considerably larger, since the change obtained by Shapiro et al. (2011), in the correction proposed by Judge et al. (2012), is about $3 \mathrm{~W} \mathrm{~m}^{-2}$. More important for the purposes of the present paper, is that, despite this quantitative difference, the trends shown by all reconstructions are qualitatively similar. In other words, irrespective of the data or the technique used for the TSI reconstruction, the TSI is always lower during the MM than during the DM by on average $0.2-0.3 \mathrm{~W} \mathrm{~m}^{-2}$ (more for the model by Shapiro et al. 2011). This appears to be a rather robust feature. If the sunspot numbers during the MM were as high as proposed by ZP15 then this difference would vanish. This contradicts all TSI reconstructions, including ones that are not built on sunspot numbers at all, although the uncertainties in the TSI reconstructions are sufficiently large that this cannot be judged to be a very stong constraint (and is not entirely independent of the other arguments provided in this paper).

\section{Conclusions}

We revisited the level of solar activity during the MM, using all the existing, both direct and indirect, datasets and evidence to show that the activity was very low, significantly lower than during the DM or the current weak solar cycle \#24. We compared the data available with two scenarios of the solar activity level during the $\mathrm{MM}$ - the low activity (L-scenario) and the high activity (H-scenario, see Sect. 2).

The results can be summarized as follows:

- We evaluated, using a conservative approach, the fraction of sunspot active days during the MM, which appeared small, implying a very low level of sunspot activity.

- We revisited the telescopic solar observations during the MM and concluded that the astronomers of the 17 th century, especially in its second half, were very unlikely to have been influenced by the religious or philosophical dogmas. This is contrary to the claim by ZP15.

- We discussed that the short gaps in the HS98 database, that were interpreted by ZP15 as deliberate omissions of sunspot records by 17 th century observers for non-scientific reasons, are caused by a technical artefact of the database compilation and do not correspond to observational lacunas.

- We pointed out outdated and erroneous information and serious methodological flaws in the analysis done by ZP15 that led them to severely overestimate the solar activity level during the MM.

- We showed that the latitudinal extent of sunspot formation also points to a very low solar activity during the MM.

- We showed that East-Asian naked-eye sunspot observations cannot be used to assess the exact level of solar activity. The existing data do not contradict to the very low activity level during the MM.

- We presented a re-analysis of several documented sources, some new to science so far, on the occurrence of lowerlatitude aurorae on Earth. We demonstrated that the MM indeed displayed very low activity also in terms of auroral sightings compared to normal periods and also to the DM.

- We compared the estimated heliospheric conditions for the two scenarios with the actual measured cosmogenic isotope data for the period around the MM. The comparison is fully consistent with the L-scenario but rejects the $\mathrm{H}$-scenario at a very high confidence level.

- We argued that the observational facts (very low sunspot activity, hemispheric asymmetry of sunspot formation, unusual differential rotation of the solar surface and the lack of the K-corona) imply a special mode of the solar dynamo during the MM, and disfavour an interpretation of the latter as a regular minimum of the centennial Gleissberg cycle.

- We discussed consequences of the MM for the solar irradiance variability, which is a crucial point for the assessment of solar variability influence on both global and regional climate (Lockwood 2012; Solanki et al. 2013).

We concluded, after careful revision of all the presently available datasets for the Maunder minimum, that solar activity was 
indeed at an exceptionally low level during that period, corresponding to a special grand minimum mode of solar dynamo. The suggestions of a moderate-to-high level of solar activity during the Maunder minimum are rejected at a high significance level.

Acknowledgements. We are grateful to Regina von Berlepsch, Potsdam, and Pierre Leich, Nürnberg, the ECHO digital heritage online, and the Swiss Electronic Library, E-lib.ch, for providing electronic versions of a number of historic publications and manuscripts. We are very grateul to Ralph Neuhäuser for pointing to the original work by Marius, and to Clara Ricken and Yori Fournier for their help with translations of original sunspot records. This work was partly done in the framework of the ReSoLVE Centre of Excellence (Academy of Finland, project No. 272157) and partly supported by the BK21 plus program through the National Research Foundation (NRF) funded by the Ministry of Education of Korea. J.M.V. acknowledges the support from the Junta de Extremadura (Research Group Grants GR10131) and from the Spanish Government (AYA2011-25945 and AYA2014-57556-P). D.S. acknowledges Project RFFI 15-02-01407. W.S. received no funding for his contribution to this paper. Support from the COST Action ES1005 TOSCA (www. tosca-cost.eu) is gratefully acknowledged.

\section{References}

Ahmad, I., Bonino, G., Castagnoli, G. C., et al. 1998, Phys. Rev. Lett., 80, 2550 Alanko-Huotari, K., Mursula, K., Usoskin, I. G., \& Kovaltsov, G. A. 2006, Sol. Phys., 238, 391

Argolus, A. 1644, Pandosion sphaericum (Patavia: Paulus Frambottus)

Arlt, R. 2008, Sol. Phys., 247, 399

Arlt, R. 2009, Sol. Phys., 255, 143

Arlt, R., \& Fröhlich, H.-E. 2012, A\&A, 543, A7

Arlt, R., Leussu, R., Giese, N., Mursula, K., \& Usoskin, I. G. 2013, MNRAS, 433,3165

Asvestari, E., \& Usoskin, I. G. 2015, Sol. Phys., submitted

Augustson, K., Brun, S., Miesch, M., \& Toomre, J. 2015, 18th Cambridge Workshop on Cool Stars, Stellar Systems and the Sun, Proc. of the Conf. held at Lowell Observatory, eds. G. van Belle \& H. C. Harris, 451

Barnard, L., Lockwood, M., Hapgood, M. A., et al. 2011, Geophys. Res. Lett., 381, L16103

Beer, J. 2000, Space Sci. Rev., 94, 53

Beer, J., Tobias, S., \& Weiss, N. 1998, Sol. Phys., 181, 237

Beer, J., McCracken, K., \& von Steiger, R. 2012, Cosmogenic Radionuclides: Theory and Applications in the Terrestrial and Space Environments (Berlin: Springer)

Berggren, A.-M., Beer, J., Possnert, G., et al. 2009, Geophys. Res. Lett., 36, L11801

Bonino, G., Castagnoli, G., Bhandari, N., \& Taricco, C. 1995, Science, 270, 1648

Brandenburg, A., Krause, F., Meinel, R., Moss, D., \& Tuominen, I. 1989, A\&A, 213,411

Brekke, A., \& Egeland, A. 1994, The northern lights: their Heritage and Science (Grøndahl Dreyer)

Brody, J. 2002, The Enigma of Sunspots: A Story of Discovery and Scientific Revolution (Edinburgh: Floris Books)

Brown, B. P., Miesch, M. S., Browning, M. K., Brun, A. S., \& Toomre, J. 2011, ApJ, 731, 69

Bushby, P. J. 2006, MNRAS, 371, 772

Caballero-Lopez, R. A., Moraal, H., McCracken, K. G., \& McDonald, F. B. 2004, J. Geophys. Res., 109, A12102

Carrasco, V. M. S., Villalba Álvarez, J., \& Vaquero, J. M. 2015, Sol. Phys., submitted [arXiv: 1502.06270]

Casas, R., \& Vaquero, J. M. 2014, Sol. Phys., 289, 79

Casas, R., Vaquero, J., \& Vazquez, M. 2006, Sol. Phys., 234, 379

Cassini, G. 1730, Memoires de l'académie royale des sciences, Depuis 1666 jusqu'á 1699, 10, 653

Chapman, A. 2004, in Transits of Venus: new views of the solar system and galaxy, IAU Colloq., 196, 3

Charbonneau, P. 2010, Liv. Rev. Sol. Phys., 7

Choudhuri, A. R., \& Karak, B. B. 2012, Phys. Rev. Lett., 109, 171103

Clark, D., \& Stephenson, F. 1978, Quart. J. Royal Astron. Soc., 19, 387

Clette, F., Svalgaard, L., Vaquero, J., \& Cliver, E. 2014, Space Sci. Rev., 186, 35

Cliver, E., Boriakoff, V., \& Bounar, K. 1998, Geophys. Res. Lett., 25, 897

Cliver, E. W., Kamide, Y., \& Ling, A. G. 2000, J. Geophys. Res., 105, 2413

Cortie, A. L. 1912, MNRAS, 73, 52

Cullen, C. 1980, Nature, 283, 427

Dalton, J. 1834, Meteorological observations and essays (Manchester: Harrison \& Crosfield)
Dasi-Espuig, M., Jiang, J., Krivova, N. A., \& Solanki, S. K. 2014, A\&A, 570, A23

De La Hire, P. 1720, Histoire de l'académie royale des sciences, 124

de Mairan, J. 1733, Traité Physique et Historique de l'aurore Boréale (Paris: Imprimerie Royale), 2nd edn., 1754

Delaygue, G., \& Bard, E. 2011, Clim. Dynamics, 36, 2201

Derham, W. 1703, Philosophical Transactions Series I, 23, 1504

Derham, W., \& Crabtrie, W. 1711, Royal Soc. London Philosoph. Transact. Ser. I, 27, 270

Diercke, A., Arlt, R., \& Denker, C. 2015, Astron. Nachr., 336, 53

Domingo, V., Ermolli, I., Fox, P., et al. 2009, Space Sci. Rev., 145, 337

Eddy, J. 1976, Science, 192, 1189

Eddy, J. 1983, Sol. Phys., 89, 195

Fara, P. 1996, J. Hist. Astron., 27, 239

Fligge, M., Solanki, S. K., \& Unruh, Y. C. 2000, A\&A, 353, 380

Frick, J. 1681, Philosophisches und Theologisches Bedencken, in welchem die vornehmste Meynungen von den Cometen vorgetragen und examiniert werden (Ulm: G.W. Kühnen)

Fritz, H. 1873, Verzeichniss Beobachteter Polarlichter (C. Gerold's Sohn.)

Fritz, H. 1881, Das Polarlicht (Leipzig, East Germany: F. A. Brockhaus)

Fröhlich, C. 2009, A\&A, 501, L27

Fröhlich, C. 2013, Space Sci. Rev., 176, 237

Galilei, G. 1613, Istoria e dimostrazioni intorno alle macchie solari (Rome: Mascardi)

Galileo, G., \& Scheiner, C. 2010, On Sunspots, translated and introduced by E. Reeves and A. van Helden (Chicago University Press)

Gastine, T., Duarte, L., \& Wicht, J. 2012, A\&A, 546, A19

Ghizaru, M., Charbonneau, P., \& Smolarkiewicz, P. K. 2010, ApJ, 715, L133

Gleissberg, W. 1977, Sterne Weltraum, 16, 229

Gleissberg, W., Damboldt, T., \& Schove, D. J. 1979, J. British Astron. Assoc., 89,440

Gómez, J. M., \& Vaquero, J. M. 2015, The Observatory, ArXiv e-prints [arXiv: 1502.6772]

Habashi, F. 2007, in Hevel, Johannes, eds. T. Hockey, V. Trimble, T. R. Williams, et al. (New York: Springer), 501

Halley, E. 1716, Phil. Trans., 29, 406

Harriot, T. 1613, Harriot papers, Vol. VIII: Spots on the Sun. (MPI for the History of Science, Library: Petworth House Archives, HMC 241 VIII; digital version)

Harrison, R. 2005, Astron. Geophys., 46, 4.31

Harvey, K., \& White, O. 1999, J. Geophys. Res., 104, 19759

Hathaway, D. H. 2010, Liv. Rev. Sol. Phys., 7, 1

Heikkilä, U., Beer, J., \& Feichter, J. 2009, Atmos. Chem. Phys., 9, 515

Heilborn, J. 1999, The Sun in Church (Harvard University Press), 366

Hevelius, J. 1647, Selenographia: sive lunae descriptio (Danzig: Hünefeld)

Hevelius, J. 1679, Machina coelestis/ 2: Rerum Uranicarum Observationes/ and 3: Altitudines videlicet Solares (Danzig: S. Reiniger)

Horiuchi, K., Ohta, A., Uchida, T., et al. 2007, Nucl. Inst. Meth. Phys. Res. B, 259,584

Horrebow, N. 1752, Tilforladelige Efterretninger om Island (Köbehhavn)

Hoyt, D. V., \& Schatten, K. H. 1995, Sol. Phys., 160, 371

Hoyt, D. V., \& Schatten, K. H. 1998, Sol. Phys., 179, 189

Hutchison, K. 1990, Isis, 81, 68

Ivanov, V. G., \& Miletsky, E. V. 2011, Sol. Phys., 268, 231

Jackson, A., Jonkers, A. R. T., \& Walker, M. R. 2000, in Astronomy, physics and chemistry of $\mathrm{H}_{3}^{+}$, Roy. Soc. London Phil. Trans. Ser. A, 358, 957

Jennings, R., \& Weiss, N. 1991, MNRAS, 252, 249

Jiang, J., Cameron, R., Schmitt, D., \& Schüssler, M. 2010, ApJ, 709, 301

Jiang, J., Cameron, R. H., Schmitt, D., \& Schüssler, M. 2011a, A\&A, 528, A82

Jiang, J., Cameron, R. H., Schmitt, D., \& Schüssler, M. 2011b, A\&A, 528, A83

Judge, P. G., Lockwood, G. W., Radick, R. R., et al. 2012, A\&A, 544, A88

Käpylä, P. J., Korpi, M. J., \& Brandenburg, A. 2009, A\&A, 500, 633

Käpylä, P. J., Mantere, M. J., \& Brandenburg, A. 2012, ApJ, 755, L22

Kopp, G. 2014, J. Space Weather Space Clim., 4, A14

Kovaltsov, G., \& Usoskin, I. 2010, Earth Planet. Sci. Lett., 291, 182

Kovaltsov, G. A., Usoskin, I. G., \& Mursula, K. 2004, Sol. Phys., 224, 95

Kovaltsov, G., Mishev, A., \& Usoskin, I. 2012, Earth Planet. Sci. Lett., 337, 114

Kozyra, J. U., Nagy, A. F., \& Slater, D. W. 1997, Rev. Geophys., 35, 155

Krivova, N., Balmaceda, L., \& Solanki, S. 2007, A\&A, 467, 335

Krivova, N. A., Solanki, S. K., Fligge, M., \& Unruh, Y. C. 2003, A\&A, 399, L1

Krivova, N. A., Vieira, L. E. A., \& Solanki, S. K. 2010, J. Geophys. Res., 115, A 12112

Küker, M., Arlt, R., \& Rüdiger, G. 1999, A\&A, 343, 977

Kr̆ivský, L., \& Pejml, K. 1988, Publ. Astron. Inst. Czech Acad. Sci., 75, 32

Legrand, J., Le Goff, M., Mazaudier, M., \& Schröder, W. 1992, Acta Geodaetica et Geophysica Hungarica, 27, 251

Legrand, J.-P., \& Simon, P. A. 1987, Annal. Geophys., 5, 161

A95, page 18 of 19 
Licht, A., Hulot, G., Gallet, Y., \& Thébault, E. 2013, Phys. Earth Planet. Inter., 224, 38

Link, F. 1964, Geofysikální Sborník, 12, 501

Link, F. 1978, Sol. Phys., 59, 175

Lockwood, M. 2010, Proc. R. Soc. London, Ser. A, 466, 303

Lockwood, M. 2012, Surveys Geophys., 33, 503

Lockwood, M. 2013, Liv. Rev. Sol. Phys., 10, 4

Lockwood, M., \& Owens, M. J. 2014, J. Geophys. Res., 119, 5193

Lockwood, M., Stamper, R., \& Wild, M. N. 1999, Nature, 399, 437

Lockwood, M., Nevanlinna, H., Barnard, L., et al. 2014a, Annal. Geophys., 32, 383

Lockwood, M., Owens, M. J., \& Barnard, L. 2014b, J. Geophys. Res., 119, 5172

Lovering, J. 1860, Mem. Amer. Acad. Arts Sci., 9, 101

Lovering, J. 1867, Mem. Amer. Acad. Arts Sci., New Ser., 9, 101

Lowe, E. 1870, Natural phenomena and chronology of the seasons (York Street, Covent Garden, London: Bell and Dadly)

Lyatsky, W., Newell, P. T., \& Hamza, A. 2001, Geophys. Res. Lett., 28, 2353

Manfredi, E. 1736, De Gnomone Meridiano Bononiensi ad Divi Petronii (Bononiae: Laeli a Vulpa), 397

Maunder, E. W. 1922, Brit. Astron. Assoc. J., 32, 140

McCracken, K., McDonald, F., Beer, J., Raisbeck, G., \& Yiou, F. 2004, J. Geophys. Res., 109, 12103

McCracken, K. G., \& Beer, J. 2014, J. Geophys. Res., 119, 2379

Moss, D., Saar, S. H., \& Sokoloff, D. 2008a, MNRAS, 388, 416

Moss, D., Sokoloff, D., Usoskin, I., \& Tutubalin, V. 2008b, Sol. Phys., 250, 221

Mossman, J. 1989, Quat. J. Roy. Astron. Soc., 30, 59

Nagovitsyn, Y. 1997, Astron. Lett., 23, 742

Nagovitsyn, Y. 2001, Geomag. Aeronom., 41, 680

Nagovitsyn, Y. A., Ivanov, V. G., Miletsky, E. V., \& Volobuev, D. M. 2004, Sol. Phys., 224, 103

Nevanlinna, H. 1995, J. Geomag. Geoelectr., 47, 953

Nevanlinna, H., \& Pulkkinen, T. I. 2001, J. Geophys. Res., 106, 8109

Newell, P. T., Sotirelis, T., Skura, J. P., Meng, C., \& Lyatsky, W. 2002, J. Geophys. Res., 107, 1305

Ogurtsov, M. G., Kocharov, G. E., \& Nagovitsyn, Y. A. 2003, Astron. Rep., 47, 517

Oldenburg, H. 1671a, Philosophical Transactions Series I, 6, 2216

Oldenburg, H. 1671b, Philosophical Transactions Series I, 6, 2250

Oldenburg, H. 1671c, Philosophical Transactions Series I, 6, 3020

Owens, M. J., \& Lockwood, M. 2012, J. Geophys. Res., 117, A04102

Owens, M. J., Crooker, N. U., \& Lockwood, M. 2011, J. Geophys. Res., 116, A04111

Owens, M. J., Usoskin, I., \& Lockwood, M. 2012, Geophys. Res. Lett., 39, L19102

Passos, D., \& Charbonneau, P. 2014, A\&A, 568, A113

Passos, D., Nandy, D., Hazra, S., \& Lopes, I. 2014, A\&A, 563, A18

Paton, J. 1959, The Observatory, 78, 219

Pipin, V. V. 1999, A\&A, 346, 295

Potgieter, M. 2013, Liv. Rev. Sol. Phys., 10, 3

Reimer, P. J., Bard, E., Bayliss, A., et al. 2013, Radiocarbon, 55, 1869

Rek, R. 2013, in Johannes Hevelius and His World, eds. R. Kremer, \&

J. Wlodarczyk (Warsaw: Polish Academy of Sciences), 81

Ribes, J., \& Nesme-Ribes, E. 1993, A\&A, 276, 549

Riccioli, J. 1653, Almagestum Novum. Tomus 1 (Frankfurt am Main: Beyer)

Riley, P., Lionello, R., Linker, J. A., et al. 2015, ApJ, 802, 105

Roth, R., \& Joos, F. 2013, Clim. Past, 9, 1879

Rubenson, R. 1882, Kl. Sven. Vetenskapsakad. Handl., 18, 1

Russell, C. T., \& McPherron, R. L. 1973, J. Geophys. Res., 78, 92

Sabine, E. 1852, Philos. Trans. R. Soc. London, 142, 103

Schaefer, B. E. 1993, ApJ, 411, 909

Scheiner, C. 1630, Rosa Ursina Sive Sol (Bracciano: Andrea Fei)

Scheiner, C. A. A. 1612, De Maculis Solaribus et de Stellis circum Jovem errantibus Accuratior Disquisitio (Augsburg: Ad insigne pinus)

Schove, D. 1955, J. Geophys. Res., 60, 127

Schove, D. 1983, Annales Geophys., 1, 391

Schrinner, M., Rädler, K.-H., Schmitt, D., Rheinhardt, M., \& Christensen, U. 2005, Astron. Nachr., 326, 245

Schrinner, M., Petitdemange, L., \& Dormy, E. 2012, ApJ, 752, 121

Schröder, R. 2002, in Die Normativität des Wirklichen, Über die Grenze zwischen Sein und Sollen, eds. T. Buchheim, R. Schönberger, W. Schweidler (Stuttgart: Klett-Cotta), 3

Schröder, W. 1992, J. Geomagn. Geoelectr., 44, 119

Schüssler, M., Schmitt, D., \& Ferriz-Mas, A. 1997, in 1st Advances in Solar Physics Euroconference: Advances in the Physics of Sunspots, eds. B. Schmieder, J. del Toro Iniesta, \& M. Vázquez (San Francisco, USA: ASP), ASP Conf. Ser., 118, 39

Shapiro, A. I., Schmutz, W., Rozanov, E., et al. 2011, A\&A, 529, A67
Sheeley, Jr., N. R. 2005, Liv. Rev. Sol. Phys., 2, 5

Silverman, S. 1992, Rev. Geophys., 30, 333

Siscoe, G. 1980, Rev. Geophys. Space Phys., 18, 647

Smith, A. M. 1985, Isis, 76, 543

Smogulecz, J., \& Schönberger, G. 1626, Sol illustratus ac propugnatus (Freiburg im Breisgau: T. Meyer)

Sokoloff, D. 2004, Sol. Phys., 224, 145

Sokoloff, D., \& Nesme-Ribes, E. 1994, A\&A, 288, 293

Solanki, S., Schüssler, M., \& Fligge, M. 2000, Nature, 408, 445

Solanki, S., Schüssler, M., \& Fligge, M. 2002, A\&A, 383, 706

Solanki, S. K., \& Krivova, N. A. 2011, Science, 334, 916

Solanki, S. K., Wenzler, T., \& Schmitt, D. 2008, A\&A, 483, 623

Solanki, S. K., Krivova, N. A., \& Haigh, J. D. 2013, ARA\&A, 51

Soon, W.-H., \& Yaskell, S. 2003, The Maunder Minimum and the Variable Sun-Earth Connection (Singapore; River Edge, U.S.A.: World Scientific)

Spörer, G. 1889, Nova Acta der Ksl. Leop.-Carol. Deutschen Akademie der Naturforscher, 53, 281

Stauning, P. 2011, Hist. Geo Space Sci., 1

Steinhilber, F., Beer, J., \& Fröhlich, C. 2009, Geophys. Res. Lett., 361, L19704

Taricco, C., Bhandari, N., Cane, D., Colombetti, P., \& Verma, N. 2006, J. Geophys. Res., 111, A08102

Thomas, S. R., Owens, M. J., Lockwood, M., \& Scott, C. J. 2014, Sol. Phys., 289,2653

Topper, D. 1999, Isis, 90, 757

Tromholt, S. 1898, Catalogue der in Norwegen bis Juni 1872 beobachteter Nordlichter (Jacob Dybwad Forlag)

Usoskin, I. G. 2013, Liv. Rev. Sol. Phys., 10, 1

Usoskin, I. G., Mursula, K., \& Kovaltsov, G. A. 2001, J. Geophys. Res., 106, 16039

Usoskin, I. G., Mursula, K., Solanki, S. K., Schüssler, M., \& Kovaltsov, G. A. 2002, J. Geophys. Res., 107, 1374

Usoskin, I. G., Alanko-Huotari, K., Kovaltsov, G. A., \& Mursula, K. 2005, J. Geophys. Res., 110, A12108

Usoskin, I. G., Solanki, S. K., Taricco, C., Bhandari, N., \& Kovaltsov, G. A. 2006, A\&A, 457, L25

Usoskin, I. G., Solanki, S. K., \& Kovaltsov, G. A. 2007, A\&A, 471, 301

Usoskin, I. G., Horiuchi, K., Solanki, S., Kovaltsov, G. A., \& Bard, E. 2009a, J. Geophys. Res., 114, A03112

Usoskin, I. G., Mursula, K., Arlt, R., \& Kovaltsov, G. A. 2009b, ApJ, 700, L154

Usoskin, I. G., Hulot, G., Gallet, Y., et al. 2014, A\&A, 562, L10

Vaquero, J. M. 2007, Adv. Space Res., 40, 929

Vaquero, J. M., \& Gallego, M. C. 2014, Adv. Space Res., 53, 1162

Vaquero, J. M., \& Trigo, R. M. 2014, Sol. Phys., 289, 803

Vaquero, J. M., \& Trigo, R. M. 2015, New Astron., 34, 120

Vaquero, J. M., \& Vázquez, M. 2009, The Sun Recorded Through History: Scientific Data Extracted from Historical Documents (Berlin: Springer), Astrophys. Space Sci. Lib., 361

Vaquero, J. M., Gallego, M. C., \& García, J. A. 2002, Geophys. Res. Lett., 29, 58

Vaquero, J. M., Gallego, M. C., Usoskin, I. G., \& Kovaltsov, G. A. 2011, ApJ, 731, L24

Vaquero, J. M., Trigo, R. M., \& Gallego, M. C. 2012, Sol. Phys., 277, 389

Vaquero, J. M., Gutiérrez-López, S., \& Szelecka, A. 2014, Adv. Space Res., 53, 1180

Vaquero, J. M., Kovaltsov, G. A., Usoskin, I. G., Carrasco, V. M. S., \& Gallego, M. C. 2015a, A\&A, 577, A71

Vaquero, J. M., Nogales, J. M., \& Sánchez-Bajo, F. 2015b, Adv. Space Res., 55, 1546

Vázquez, M., Vaquero, J. M., \& Gallego, M. C. 2014, Sol. Phys., 289, 1843

Vieira, L. E. A., Solanki, S. K., Krivova, N. A., \& Usoskin, I. 2011, A\&A, 531, A6

Vitinskii, I. I. 1978, Sol. Phys., 57, 475

Vitinsky, Y., Kopecky, M., \& Kuklin, G. 1986, Statistics of Sunspot Activity (in Russian) (Moscow: Nauka)

Volobuev, D. 2004, Sol. Phys., 224, 387

Wang, Y.-M., \& Sheeley, N. R. 2002, J. Geophys. Res., 107, 1302

Wang, Y.-M., Lean, J., \& Sheeley Jr, N. 2005, ApJ, 625, 522

Willis, D., Davda, V., \& Stephenson, F. 1996, Quart. J. Roy. Astron. Soc., 37, 189

Wittmann, A. 1978, A\&A, 66, 93

Wolf, R. 1856, Vierteljahrsschrift der Naturforschenden Gesellschaft in Zürich, 1,151

Yau, K. K. C., \& Stephenson, F. R. 1988, Quat. J. R. Astron. Soc., 29, 175

Yau, K. K. C., Stephenson, F. R., \& Willis, D. M. 1995, A Catalogue of Auroral Observations from China, Korea and Japan (193 BCAD 1770), Tech. Rep. RALTR95073, Rutherford Appleton Laboratory

Zhang, Z. W. 1985, J. British Astron. Assoc., 95, 205

Zolotova, N. V., \& Ponyavin, D. I. 2015, ApJ, 800, 42 